\title{
Collocation Methods for Boundary Value Problems on 'Long' Intervals
}

\author{
By Peter A. Markowich* and Christian A. Ringhofer**
}

\begin{abstract}
This paper deals with the numerical solution of boundary value problems of ordinary differential equations posed on infinite intervals. We cut the infinite interval at a finite, large enough point and insert additional, so-called asymptotic boundary conditions at the far (right) end and then solve the resulting two-point boundary value problem by an $A$-stable symmetric collocation method. Problems arise, because standard theory predicts the use of many grid points as the length of the interval increases. Using the exponential decay of the 'infinite' solution, an 'asymptotic' a priori mesh-size sequence which increases exponentially, and which therefore only employs a reasonable number of meshpoints, is developed and stability, as the length of the interval tends to infinity, is shown. We also show that the condition number of the collocation equations is asymptotically proportional to the number of meshpoints employed when using this exponentially graded mesh. Using $k$-stage collocation at Gaussian points and requiring an accuracy $O(\varepsilon)$ at the knots implies that the number of meshpoints is $O\left(\varepsilon^{-1 / 2 k}\right)$ as $\varepsilon \rightarrow 0$.
\end{abstract}

1. Introduction. In this paper the numerical solution of boundary value problems on infinite intervals of the form

$$
\begin{gathered}
y^{\prime}=t^{\alpha} f(t, y), \quad 1 \leqslant t<\infty, \alpha \geqslant 0, \\
b(y(1))=0, \\
y \in C([1, \infty]): \Leftrightarrow y \in C([1, \infty)) \text { and } \lim _{t \rightarrow \infty} y(t)=y(\infty) \text { is finite }
\end{gathered}
$$

is considered. Here $f: R^{n+1} \rightarrow R^{n}, b: R^{n} \rightarrow R^{k}$, where generally $k<n$ holds because (1.3) furnishes another set of boundary conditions. $f$ fulfills certain continuity properties at infinity which will be defined later. We assume that the Jacobian $\partial f(\infty, y(\infty)) / \partial y$ has no eigenvalue on the imaginary axis.

For $\alpha>-1$, Eq. (1.1) has a singularity of the second kind of rank $\alpha+1$ at $t=\infty$. We disregard the practically unimportant case $-1<\alpha<0$ in the sequel.

Problems of this kind often occur in fluid dynamics (boundary layer theory), quantum mechanics and electronics. For applications see Markowich [12], [13], de Hoog and Weiss [7], McLeod [15] and Schneider [19].

Received March 30, 1981; revised December 7, 1981 and April 23, 1982.

1980 Mathematics Subject Classification. Primary 34B15, 34C05, 34D05, 34A10, 34A11.

Key words and phrases. Nonlinear boundary value problems, singular points, asymptotic properties, difference equations, stability of difference equations.

*Sponsored by the United States Army under Contract No. DAAG29-80-C-0041 and the Austrian Ministry for Science and Research. This material is based upon work supported by the National Science Foundation under Grant No. MCS-7927062. Current address: Institut fuer Angewandte Mathematik, Technische Universität Wien, Gusshausstrasse 27-29, A-1040 Wien, Austria.

**Supported by a Research Grant from the Austrian Forschungsförderungsfond. Current address: University of Wisconsin-Madison, Mathematics Research Center, 610 Walnut Street, Madison, WI 53706. 
For the numerical solution we proceed as follows. First the infinite interval is substituted by a finite but large interval and $n-k$ additional so-called asymptotic boundary conditions, which reflect the asymptotic behavior of the solution $y$, are imposed at the right (far) endpoint $T$. We obtain a regular two-point boundary value problem of the form

$$
\begin{gathered}
x^{\prime}=t^{\alpha} f(t, x), \quad 1 \leqslant t \leqslant T, \\
b(x(1))=0, \\
S(T) x(T)=\sigma(T) .
\end{gathered}
$$

The condition (1.6) has to be chosen such that

$$
\|y-x\|_{[1, T]} \rightarrow 0 \text { as } T \rightarrow \infty
$$

holds, and its construction is described in de Hoog and Weiss [8], Lentini and Keller [10] and Markowich [13].

The two-point boundary value problem (1.4), (1.5), (1.6) now has to be solved by an appropriate numerical method, for example by $A$-stable, symmetric collocation methods whose simplest member is the Box-scheme:

$$
\begin{gathered}
\frac{x_{i+1}-x_{i}}{h_{i}}=t_{i+1 / 2}^{\alpha} f\left(t_{i+1 / 2}, \frac{1}{2}\left(x_{i+1}+x_{i}\right)\right), \quad i=0(1)(N-1), \\
b\left(x_{0}\right)=0, \\
S(T) x_{n}=\sigma(T),
\end{gathered}
$$

where $t_{0}=1<t_{1}<\cdots<t_{N-1}<t_{N}=T, t_{i+1}=t_{i}+h_{i}, t_{i+1 / 2}=t_{i}+h_{i} / 2$ holds.

It is clear that the mesh-size selection is, especially for these problems, very important since the amount of labor will be very large for long intervals and bad (too small) mesh-size choices. We do not assume a relation of the form

$$
\max _{i} h_{i} / \min _{i} h_{i} \leqslant \text { const }
$$

and do not formulate convergence estimates in terms of $\max _{i} h_{i}$ as the standard theory of collocation methods does (see Weiss [20]). Too many meshpoints would be employed in order to admit a given bound for the global error. Codes which employ adaptive mesh refinement (see Lentini and Pereyra [11] and Ascher, Christiansen and Russell [1]) solve first with a coarse grid in order to do local error estimation. Therefore it is important to know a priori which mesh-size distribution is appropriate.

In this paper we use the asymptotic form of the solution of (1.1), (1.2), (1.3) in order to construct an asymptotic a priori mesh by equidistributing the local discretization error.

It turns out that mesh sizes which increase exponentially can be used since our assumptions guarantee that $y(t) \rightarrow y(\infty)$ exponentially. For a $k$-stage collocation method at Gaussian points it will be shown that the number of grid points which is necessary in order to achieve a total accuracy $O(\varepsilon)$ (total accuracy refers to the difference between the 'infinite' solution $y\left(t_{i}\right)$ and the discrete approximation $x_{i}$ ) equals $O\left(\varepsilon^{-1 / 2 k}\right)$. For this a suitable $T=T(\varepsilon)$ will be taken. Stability (as $\varepsilon \rightarrow 0$, $T(\varepsilon) \rightarrow \infty)$ holds when using this equidistributing mesh. The Newton procedure for solving the collocation equations with these exponentially increasing stepsizes converges quadratically from a domain of starting values which does not shrink as 
$\varepsilon \rightarrow 0$. We also show that the condition number of the (linear) collocation equations (using Gaussian points) is $O\left(\varepsilon^{-1 / 2 k}\right)$, so that the linear system can be safely solved by Gaussian elimination with partial pivoting.

Of course, no fully implicit difference scheme (like the implicit Euler scheme) should be used for the integration of (1.4), (1.5), (1.6) if the fundamental matrix of the linearized problem (1.4) contains exponentially increasing columns which are scaled down by the boundary condition (1.6). This would cause instabilities during the integration when using large mesh sizes.

Another way to solve problems of the kind (1.1), (1.2), (1.3) is to transform the 'infinite' problem by a transformation $t=s^{-\beta}, \beta>0$ to the interval $[0,1]$ and to employ difference methods on the unit interval. Methods of this kind have been investigated by de Hoog and Weiss [6]. This way of proceeding has the disadvantage that a singular problem (the right-hand side of the equation is not defined in $s=0$ ) has to be solved, and therefore the obtained convergence estimates are not very strong. Another disadvantage is that many physical problems are actually posed on an infinite interval (for example in boundary layer theory) such that a 'direct' solution is desirable.

We remark that there is a close connection to singular perturbation problems since the transformation $s=(t-1) /(T-1), \mu=1 /(T-1)$ takes $(1.4),(1.5),(1.6)$ into

$$
\begin{gathered}
\mu^{\alpha+1} z^{\prime}(s)=(s+\mu)^{\alpha} f\left(\frac{s+\mu}{\mu}, z(s)\right), \quad 0 \leqslant s \leqslant 1, \alpha \geqslant 0, \\
b(z(0))=0, \\
S\left(\frac{1}{\mu}+1\right) z(1)=\sigma\left(\frac{1}{\mu}+1\right) .
\end{gathered}
$$

(Note that $\lim _{\mu \rightarrow 0} f((s+\mu) / \mu, z)=f(\infty, z)$.)

The already developed mesh-size sequences for singular perturbation problems cannot be applied without reconsideration since the linearization of the right-hand side of (1.12) does not generally have a series expansion in powers of $\mu$ uniformly in $0 \leqslant s \leqslant 1$ (see Ringhofer [16]), since for most practical problems

$$
\frac{\partial f}{\partial y}(t, y) \sim \sum_{i=0}^{\infty} A_{i}(y) t^{-i}, \quad t \rightarrow \infty,
$$

holds.

Recently Ascher and Weiss [2] came up with a mesh-size sequence for linear constant coefficient singular perturbation problems $(\alpha=0)$ (also by equidistribution) which is equivalent to ours, and which they use within the boundary layers of thickness $O(\mu|\ln \mu|$ ) (where the solution decays exponentially). Outside the layer they use a coarse mesh just fine enough to approximate the solution of the reduced problem $(\mu=0)$ well.

This paper is organized as follows. Section 2 gives a short summary of the theory of boundary value problems on infinite intervals and their 'finite' approximation, in Section 3 step-size sequences are developed for the midpoint rule for scalar initial value problems, Section 4 deals with the midpoint rule for linear boundary value problems and in Section 5 nonlinear problems are dealt with. Higher order collocation methods are analyzed in Section 6 and computations are reported in Section 7. 
2. Boundary Value Problems on Infinite Intervals and Their Approximation by 'Finite' Interval Problems-A Summary. We consider boundary value problems on infinite intervals of the following form

$$
\begin{gathered}
y^{\prime}=t^{\alpha} A(t) y+t^{\alpha} f(t), \quad 1 \leqslant t<\infty, \alpha \geqslant 0, \\
B y(1)=\beta, \\
y \in C([1, \infty]),
\end{gathered}
$$

where the $n \times n$-matrix $A \in C([1, \infty])$ and $f \in C([1, \infty]) . B$ is a matrix whose rank is (in general) less than $n$ since (2.3) furnishes another set of boundary conditions.

Let us first consider the case where $A$ is constant. $A$ has the Jordan form $J$ obtained by

$$
A=E J E^{-1} .
$$

We assume that $J$ has the block form

$$
\left.J=\left[\begin{array}{ccc}
J^{+} & 0 \\
\hdashline-1 & 0 \\
r_{+} & \underbrace{J^{-}}_{r_{-}}
\end{array}\right]\right\} r_{+}
$$

where the $r_{+} r_{+}$-matrix $J^{+}$has only eigenvalues with positive real parts and the $r_{-}$ $r_{-}$-matrix $J^{-}$has only eigenvalues with negative real parts. Eigenvalues on the imaginary axis will be excluded for the following. The diagonal projections $D_{+}, D_{-}$ are defined by

$$
\begin{aligned}
& D_{+}=\left[\begin{array}{ccc}
I_{r_{+}} & 0 \\
\hdashline 0 & 1 & 0
\end{array}\right], \\
& D_{-}=\left[\begin{array}{cc}
0 & 0 \\
\hdashline 0 & I_{r_{-}}
\end{array}\right] .
\end{aligned}
$$

The general solution of (2.1) (with $A(t) \equiv A$ ) and (2.3) can now be written as

$$
y(t)=E \phi(t)\left[\begin{array}{c}
0 \\
I_{r_{-}}
\end{array}\right] \xi+E\left(H E^{-1} f\right)(t), \quad \xi \in C^{r_{-}},
$$

where

$$
\phi(t)=\exp \left(\frac{J}{\alpha+1} t^{\alpha+1}\right)
$$

is the fundamental matrix of the transformed problem

$$
u^{\prime}=t^{\alpha} J u+t^{\alpha} E^{-1} f(t) .
$$

$E u=y$ holds and $\left(H E^{-1} f\right)(t)$ is a suitable particular solution of $(2.10)$ which can be taken as

$$
(H g)(t)=\phi(t) \int_{\infty}^{t} D_{+} \phi^{-1}(s) s^{\alpha} g(s) d s+\phi(t) \int_{\gamma}^{t} D_{-} \phi^{-1}(s) s^{\alpha} g(s) d s
$$

for some $\gamma \geqslant 1$. This operator has been analyzed by de Hoog and Weiss [7], [8] and Markowich [12].

$H$ has the following properties
$(2.12)(\mathrm{a})$
$H: C([\gamma, \infty]) \rightarrow C([\gamma, \infty])$,
$\|H\|_{[\gamma, \infty]} \leqslant c$, 
where $\|\cdot\|_{[\gamma, \infty]}$ denotes the max-norm on $[\gamma, \infty]$ respectively the associated operator-norm. The constant $c$ is independent of $\gamma$.

Markowich [12] has shown that if

$$
\begin{aligned}
& f(t)=F(t) \exp \left(-\frac{\theta}{\alpha+1} t^{\alpha+1}\right), \\
& \quad F \in L_{\infty}([1, \infty]) \cap C([1, \infty)), \theta>\lambda_{\min }>0
\end{aligned}
$$

holds where $\lambda_{\min }$ is the smallest modulus of the real parts of the eigenvalues of $A$ which are in the left half-plane, then

$$
\begin{aligned}
\|y(t)\| & \leqslant \operatorname{const}\left(\|F\|_{[1, \infty]}+\|\xi\|\right)\left\|\phi(t)\left[\begin{array}{c}
0 \\
I_{r_{-}}
\end{array}\right]\right\| \\
& \leqslant \operatorname{const}\left(\|F\|_{[1, \infty]}+\|\xi\|\right) \exp \left(-\frac{\left(\lambda_{\min }-\delta\right)}{\alpha+1} t^{\alpha+1}\right), \quad t \geqslant \bar{t},
\end{aligned}
$$

holds. $0 \leqslant \delta<\lambda_{\min }$ and $\delta \rightarrow 0$ as $\bar{t} \rightarrow \infty$ hold.

The boundary value problem (2.1), (2.2), (2.3) with $A(t) \equiv A$ is uniquely soluble for all $\beta \in R^{r_{-}}, f \in C([1, \infty])$ if and only if the $r_{-} \times r_{-}$-matrix

$$
B E \phi(1)\left[\begin{array}{c}
0 \\
I_{r_{-}}
\end{array}\right] \text {is nonsingular. }
$$

Here $B$ is assumed to be an $r_{-} \times n$-matrix. So the continuity requirement (2.3) furnishes $r_{+}$linearly independent boundary conditions. (2.12), (2.15) imply that $\|\xi\| \leqslant \operatorname{const}\left(\|\beta\|+\|F\|_{[1, \infty]}\right)$ holds.

The variable coefficient case $A(t) Z A$ is treated by a perturbation approach (see de Hoog and Weiss [7], [8] and Markowich [12], [13]). $A(\infty)$ now plays the role of $A$. We assume that $A(\infty)$ has the Jordan form $J$ given by (2.5). Then we can show (see de Hoog and Weiss [7], [8]) that

$$
y(t)=E \psi_{-}(t) \xi+E \psi\left(E^{-1} f\right)(t), \quad \xi \in C^{r_{-}},
$$

where $\psi_{-}(t)$ is an $n \times r_{-}$-matrix defined by

$$
\psi_{-}(\cdot)=\left(I-H\left(E^{-1} A(\cdot) E-J\right)\right)^{-1} \phi(\cdot)\left[\begin{array}{c}
0 \\
I_{r_{-}}
\end{array}\right] \in C([\gamma, \infty])
$$

for $\gamma$ sufficiently large. For $t \in[1, \gamma], \psi_{-}(t)$ can be continuously extended. $E \psi\left(E^{-1} f\right)$ is a suitable particular solution of (2.1). The boundary value problem (2.1), (2.2), (2.3) is uniquely soluble for every $\beta \in R^{r_{-}}, f \in C([1, \infty])$ if and only if the $r_{-} \times r_{-}-$ matrix

$$
B E \psi_{-}(1) \text { is nonsingular. }
$$

Markowich [12] proved the estimate

$$
\|y(t)\| \leqslant \operatorname{const}\left(\|F\|_{[1, \infty]}+\|\beta\|\right) \exp \left(-\frac{\left(\lambda_{\min }-\delta\right)}{\alpha+1} t^{\alpha+1}\right), \quad t \geqslant \bar{t} .
$$

Now we briefly consider nonlinear problems of the form (1.1), (1.2), (1.3). From (1.1), (1.3) we conclude that

$$
f(\infty, y(\infty))=0
$$

has to hold. We assume that the roots $y(\infty)$ of (2.20) are isolated and take one particular root $y^{*}(\infty)$ for the following. Moreover, $f\left(t, y^{*}(\infty)\right)$ shall fulfill (2.13). 
Defining

$$
C_{\kappa}(\bar{t}, a)=\left\{(t, y) \in R^{n+1} \mid t \geqslant \bar{t},\left\|y-y^{*}(\infty)\right\| \leqslant \kappa\right\},
$$

we assume that $f, f_{y} \in C_{\text {lip }}\left(C_{\kappa}\left(1, y^{*}(\infty)\right)\right)$ for a sufficiently large $\kappa$, We also assume that the boundary value problem (1.1), (1.2), (1.3) has an isolated solution, i.e. the linearized problem is nonsingular.

Now let $J$ be the Jordan form of $f_{y}\left(\infty, y^{*}(\infty)\right)$ obtained by

$$
f_{y}\left(\infty, y^{*}(\infty)\right)=E J E^{-1} \text {, }
$$

and let $J$ fulfill (2.5) such that $D_{+}, D_{-}$, are defined as in (2.6), (2.7). Then $\lambda_{\min }$ is defined for $J$ as above, and $\phi(t), \psi_{-}(t)$ are as in (2.9), (2.17) with $f_{y}\left(t, y^{*}(\infty)\right)$ substituted for $A(t)$. Markowich [12] showed that

$$
\left\|y(t)-y^{*}(\infty)\right\| \leqslant \text { const }\left\|\psi_{-}(t)\right\| \leqslant \text { const } \exp \left(-\frac{\left(\lambda_{\min }-\delta\right)}{\alpha+1} t^{\alpha+1}\right) .
$$

The isolatedness of $y$ now implies that

$$
\frac{\partial b}{\partial y}(y(1)) \psi_{-}(1)
$$

is nonsingular. More information on the analysis of these problems can be found in the above cited references and in Lentini and Keller [10].

We want to approximate the 'infinite' problem (2.1), (2.2), (2.3) by 'finite' two-point boundary value problems of the form

$$
\begin{gathered}
x^{\prime}=t^{\alpha} A(t) x+t^{\alpha} f(t), \quad 1 \leqslant t \leqslant T, T \gg 1, \\
B x(1)=\beta, \\
S(T) x(T)=\sigma(T) .
\end{gathered}
$$

Since (2.24) is a boundary condition of rank $r_{-}$, we assume the $S(T)$ is an $r_{+} \times n$-matrix. The question that arises immediately is how to construct an asymptotic boundary condition $S(T)$ such that

$$
\|y-x\|_{[1, T]} \rightarrow 0 \text { as } T \rightarrow \infty,
$$

and the order of convergence should be as fast as possible.

A complete theory of this kind can be found in de Hoog and Weiss [7] and Markowich [13], and therefore we only give excerpts which will be needed in the sequel. The basic idea is that the boundary condition (2.25) has to scale down all solution components of (2.23) which do not decay exponentially.

We assume that (2.18) holds. A possible choice is

$$
S \equiv S(T)=\left[I_{r_{+}}, 0\right] E^{-1}, \quad \sigma(T)=0 .
$$

It has been shown in the above cited references that this boundary condition implies convergence in the sense of (2.26) and that for general $\sigma(T)$

$$
\|y-x\|_{[1, T]} \leqslant \text { const }\|S y(T)-\sigma(T)\|
$$

holds. In general the admissibility conditions for a boundary condition $S(T)$ are

$$
\begin{gathered}
\|S(T)\| \leqslant \text { const, } \quad T \rightarrow \infty, \\
\left\|\left(S(T) E\left[\begin{array}{c}
I_{r_{+}} \\
0
\end{array}\right]\right)^{-1}\right\| \leqslant \text { const, } \quad T \rightarrow \infty .
\end{gathered}
$$


Then (2.28) holds for the unique solution $x$ of the 'finite' problem if $T$ is sufficiently large. $\sigma(T) \equiv 0$ is a natural choice for linear problems.

If $f(t)$ fulfills (2.13) an estimate for the order of convergence is given by the right-hand side of (2.19). Moreover, it has been shown that the choice (2.27) is optimal in the sense that the actual order of convergence exceeds (2.19) for homogeneous problems.

The condition (2.25) with $S$ fulfilling (2.29), (2.30) and $\sigma(T)=S(T) y^{*}(\infty)$ can also be used for nonlinear problems of the form (1.1), (1.2), (1.3) if the above stated assumptions on $f(t, y)$ and the solution $y$ hold. (2.28) still holds for nonlinear problems. So we obtain

$$
\|y-x\|_{[1, T]} \leqslant \text { const }\|S(T)\| \cdot \varphi \cdot \exp \left(-\frac{\left(\lambda_{\min }-\delta\right)}{\alpha+1} T^{\alpha+1}\right)
$$

where

$$
\varphi=\max _{t \in[1, \infty]}\left\|\left(y(t)-y^{*}(\infty)\right) \exp \left(\frac{\lambda_{\min }-\delta}{\alpha+1} t^{\alpha+1}\right)\right\|<\infty
$$

has been set. The constant in (2.31) is an upper bound for the norm of the inverse of the linearization of (1.4), (1.5), (1.6) at $y$. In order to get $\|y-x\|_{[1, T]} \leqslant$ const $\|S(T)\| \varepsilon$, we choose

$$
T=T(\varepsilon)=\left(\frac{\alpha+1}{\lambda_{\min }-\delta} \ln \frac{\varphi}{\varepsilon}\right)^{1 /(\alpha+1)}, \quad \varepsilon \ll 1 .
$$

The advantage of choosing $\varphi$ as in (2.32) is that

$$
\left\|y(T(\varepsilon))-y^{*}(\infty)\right\| \leqslant \varepsilon
$$

holds, such that $\varepsilon$ controls how close $y(T)$ is to its asymptotic state $y^{*}(\infty)$, and then the constant in (2.31) does not (at least for linear problems) depend on $y$. However, if no estimate on $\varphi$ is available, it can be incorporated into $\varepsilon$ by setting $\bar{\varepsilon}=\varepsilon / \varphi$.

If the function $f$, which sets up the differential equation (1.1) is independent of $t$, a stronger estimate holds:

$$
\|x-y\|_{[1, T]} \leqslant \text { const }\|S(T)\| \varphi \cdot \exp \left(-\frac{2\left(\lambda_{\min }-\delta\right)}{\alpha+1} T^{\alpha+1}\right)
$$

if $S$ fulfills (2.27) and $\sigma(T)=S(T) y^{*}(\infty)$. In this case we set

$$
T=T(\varepsilon)=\frac{1}{2}\left(\frac{\alpha+1}{\lambda_{\min }-\delta} \ln \frac{\varphi}{\varepsilon}\right)^{1 / \alpha+1},
$$

and $\|x-y\|_{[1, T(\varepsilon)]} \leqslant$ const $\varepsilon$ holds.

For constant coefficient problems ( $A$ is a constant matrix) we can determine $\delta$ explicitly. In this case we obtain from Markowich [13] a sharper bound for the approximation error than (2.31):

$$
\|y-x\|_{[1, T]} \leqslant \text { const }\|S(T)\| \psi \cdot T^{(\alpha+1)(r-1)} \exp \left(-\frac{\lambda_{\min }}{\alpha+1} T^{\alpha+1}\right),
$$

where $r$ is the dimension of the largest Jordan block of $A$ with an eigenvalue with real part $-\lambda_{\min }$ and

$$
\psi=\max _{t \in[1, \infty]}\left\|y(t) t^{-(\alpha+1)(r-1)} \exp \left(\frac{\lambda_{\min }}{\alpha+1} t^{\alpha+1}\right)\right\|
$$


holds. Inserting (2.33) (with $\psi$ substituted for $\varphi$ ) into the right-hand side of (2.37) and requiring that $\delta \leqslant \lambda_{\min } / 2$ and $\|y-x\|_{[1, T(\varepsilon)]} \leqslant$ const $\|S(T(\varepsilon))\| \cdot \varepsilon$ hold gives a lower bound for $\delta$ depending on $\varepsilon$

$$
\delta \geqslant \frac{(r-1) \lambda_{\min }}{\ln \psi / \varepsilon} \ln \left|\frac{\lambda_{\min }}{2(\alpha+1) \ln \psi / \varepsilon}\right|=\bar{\delta}(\varepsilon) .
$$

Obviously $\bar{\delta}(\varepsilon) \rightarrow 0$ as $\varepsilon \rightarrow 0$ such that $\bar{\delta}(\varepsilon) \leqslant \lambda_{\min } / 2$ holds for $\varepsilon$ sufficiently small. $\delta=\bar{\delta}(\varepsilon)$ can also be used for nonlinear problems if $f$ does not depend on $t$. For variable coefficient problems whose coefficient matrix has an asymptotic series expansion as $t \rightarrow \infty$, a similar approach can be used for the determination of $\delta$ by using the estimates given in Markowich [12].

3. The Scalar Case. In this section we treat the simplest case, namely scalar initial value problems. The aim is to construct step-size sequences for the Box-scheme such that the global error is less than $O(\varepsilon)$ on $[1, T(\varepsilon)]$. These step-size sequences will be used for the general boundary value problem case.

We consider

$$
\begin{gathered}
y^{\prime}=-\lambda t^{\alpha} y+t^{\alpha} f(t), \quad 1 \leqslant t<\infty, \alpha \geqslant 0, \\
y(1)=\bar{y},
\end{gathered}
$$

where $\lambda=\lambda_{1}+i \lambda_{2}$ may vary in a compact subset $\Omega$ of $\{z \in \mathbf{C} \mid \operatorname{Re} z>0\}$.

The Box- (or centered Euler) scheme for (3.1), (3.2) has the form

$$
\frac{y_{i+1}-y_{i}}{h_{i}}=-\frac{\lambda}{2} t_{i+1 / 2}^{\alpha}\left(y_{i+1}+y_{i}\right)+t_{i+1 / 2}^{\alpha} f_{i+1 / 2}, \quad i \geqslant 0, y_{0}=\bar{y},
$$

where for $h_{i}>0$

$$
\begin{gathered}
t_{0}=1, \quad t_{i+1}=t_{i}+h_{i}, \quad t_{i+1 / 2}=t_{i}+\frac{h_{i}}{2}, \quad i \geqslant 0 \\
f_{i+1 / 2}=f\left(t_{i+1 / 2}\right)
\end{gathered}
$$

holds.

We define

$$
\begin{gathered}
Y_{n, m}(\lambda, h)=\prod_{j=n}^{m} \frac{1-\frac{\lambda}{2} h_{j} t_{j+1 / 2}^{\alpha}}{1+\frac{\lambda}{2} h_{j} t_{j+1 / 2}^{\alpha}}, \quad n \leqslant m ; \\
Y_{n+1, n}=1, \quad n \geqslant-1,
\end{gathered}
$$

and for a sequence of complex numbers $z=\left(z_{j+1 / 2}\right)_{j=1}^{i-1}$

$$
\left(H_{-}\left(\lambda, t_{I}, h\right) z\right)_{i}=\sum_{j=I}^{i-1} \frac{h_{j} t_{j+1 / 2}^{\alpha} z_{j+1 / 2}}{1+\frac{\lambda}{2} h_{j} t_{j+1 / 2}^{\alpha}} Y_{j+1, i-1}(\lambda, h), \quad i \geqslant I+1,
$$

and $\left(H_{-}\left(\lambda, t_{I}, h\right) z\right)_{I}=0$ where $h=\left(h_{j}\right)_{j=0}^{i-1}$ is the sequence of stepsizes.

Using these definitions, the solution of (3.4) can be written as

$$
y_{i}=Y_{0, i-1}(\lambda, h) \bar{y}+\left(H_{-}\left(\lambda, t_{0}, h\right) f\right)_{i},
$$

where $f=\left(f_{j+1 / 2}\right)_{j=0}^{i-1}$ has been set. 
The local discretization error $t_{i+1 / 2}^{\alpha} l_{i+1 / 2}$ of the difference scheme (3.3) is defined as

$$
\begin{aligned}
t_{i+1 / 2}^{\alpha} l_{i+1 / 2}= & \frac{y\left(t_{i+1}\right)-y\left(t_{i}\right)}{h_{i}}+\frac{\lambda}{2} t_{i+1 / 2}^{\alpha}\left(y\left(t_{i+1}\right)+y\left(t_{i}\right)\right) \\
& -t_{i+1 / 2}^{\alpha} f_{i+1 / 2}, \quad i \geqslant 0,
\end{aligned}
$$

where $y$ is the solution of (3.1), (3.2).

The global error

$$
e_{i}=y\left(t_{i}\right)-y_{i}
$$

satisfies the difference equation

$$
\frac{e_{i+1}-e_{i}}{h_{i}}=-\frac{\lambda}{2} t_{1+1 / 2}^{\alpha}\left(e_{i+1}+e_{i}\right)+t_{i+1 / 2}^{\alpha} l_{i+1 / 2}, \quad i \geqslant 0, e_{0}=0,
$$

and therefore has the solution

$$
e_{i}=\left(H_{-}\left(\lambda, t_{0}, h\right) l\right)_{i}
$$

with $l=\left(l_{j+1 / 2}\right)_{j=0}^{i-1}$.

In order to estimate the right-hand side of (3.10), we need the following

LEMMA 3.1. Let $x_{\kappa}$ for $\kappa=n, n+1, \ldots, m$, be complex numbers with $\operatorname{Re} x_{\kappa}>0$ and $\operatorname{Im} x_{\kappa} / \operatorname{Re} x_{\kappa} \leqslant x=$ const. Then setting $\prod_{l=i+1}^{i} a_{i}=0$ for $i \geqslant 1, a_{i} \in \mathbf{C}$ :

$$
\begin{gathered}
\sum_{\kappa=n}^{m} \frac{\left|x_{\kappa}\right|}{\left|1+x_{\kappa}\right|^{2}} \prod_{l=\kappa+1}^{m} \frac{\left|1-x_{l}\right|}{\left|1+x_{l}\right|} \leqslant \frac{1}{2} \sqrt{1+x^{2}}, \\
\sum_{\kappa=n}^{m} \frac{\left|x_{\kappa}\right|}{\left|1+x_{\kappa}\right|^{2}} \prod_{l=n}^{\kappa-1} \frac{\left|1-x_{l}\right|}{\left|1+x_{l}\right|} \leqslant \frac{1}{2} \sqrt{1+x^{2}}
\end{gathered}
$$

holds.

Proof. An easy calculation gives

$$
\frac{\left|x_{\kappa}\right|}{\left|1+x_{\kappa}\right|^{2}} \leqslant \frac{1}{2} \sqrt{1+x^{2}}\left(1-\frac{\left|1-x_{\kappa}\right|}{\left|1-x_{\kappa}\right|}\right) \text {. }
$$

Substitution into the right-hand sides of (3.11), (3.12) yields telescoping sums. Application of Lemma 3.1 immediately gives

Lemma 3.2. Let $f=\left(f_{j+1 / 2}\right)_{j=I}^{i-1}$. Then for every sequence $h=\left(h_{j}\right)_{j=I}^{i-1}$ with $h_{j}>0$

$$
\left|\left(H_{-}\left(\lambda, t_{I}, h\right) f\right)_{i}\right| \leqslant \text { const } \max _{j=I(1)(i-1)}\left[\left|f_{j+1 / 2}\right|\left(1+\frac{|\lambda|}{2} h_{j} t_{j+1 / 2}^{\alpha}\right)\right]
$$

holds for $i>I$ uniformly for $\lambda \in \Omega$.

Proof.

$$
\begin{aligned}
\left|\left(H_{-}\left(\lambda, t_{I}, h\right) f\right)_{i}\right| \leqslant & \text { const } \max _{j=I(1)(i-1)}\left(\left|f_{j+1 / 2}\right|\left(1+\frac{|\lambda|}{2} h_{j} t_{j+1 / 2}^{\alpha}\right)\right) \\
& \times \sum_{k=I}^{i-1} \frac{h_{k} t_{k+1 / 2}^{\alpha}}{\left|1+\frac{1}{2} h_{k} t_{k+1 / 2}^{\alpha}\right|^{2}}\left|Y_{k+1, i-1}(\lambda, h)\right|,
\end{aligned}
$$

and application of (3.11) yields (3.13). 
We get from (3.10)

$$
\left|e_{i}\right| \leqslant \text { const } \max _{j=0(1)(i-1)}\left[\left|l_{j+1 / 2}\right|\left(1+\frac{|\lambda|}{2} h_{j} t_{j+1 / 2}^{\alpha}\right)\right] .
$$

For the following we assume that

$$
f(t)=F(t) \exp \left(-\frac{\theta}{\alpha+1} t^{\alpha+1}\right), \quad \theta>\lambda_{1},
$$

holds where $F, F^{\prime}, F^{\prime \prime} \in C([1, \infty)) \cap L_{\infty}([1, \infty])$.

Markowich [12] shows that (3.15)(a) implies

$$
|y(t)| \leqslant c_{1}(\lambda)\left(\|F\|_{[1, \infty]}+|\bar{y}|\right) \exp \left(-\frac{\lambda_{1}}{\alpha+1} t^{\alpha+1}\right)
$$

where $c_{1}(\lambda)$ is bounded for $\lambda \in \Omega$.

A straightforward calculation gives

$$
\left|l_{j+1 / 2}\right| \leqslant \text { const } h_{j}^{2}\left[\frac{1}{t_{j+1 / 2}^{\alpha}}\left\|y^{\prime \prime \prime}\right\|_{\left[t_{j}, t_{j+1}\right]}+|\lambda|\left\|y^{\prime \prime}\right\|_{\left[t_{j}, t_{j+1}\right]}\right] \text {. }
$$

Differentiating (3.1) and using (3.15)(b) yields

$$
\begin{aligned}
\left|l_{j+1 / 2}\right| \leqslant & c_{2}(\lambda) h_{j}^{2}\left(\sum_{\kappa=0}^{2}\left\|F^{(\kappa)}\right\|_{[1, \infty]}+|\bar{y}|\right) t_{j}^{2 \alpha} \\
& \times \exp \left(-\frac{\lambda_{1}}{\alpha+1} t_{j}^{\alpha+1}\right), \quad t_{j} \geqslant t_{(1)}(\lambda),
\end{aligned}
$$

where the function $t^{2 \alpha} \exp \left(-\left(\lambda_{1} /(\alpha+1)\right) t^{\alpha+1}\right)$ takes its maximum over $[1, \infty]$ at $t_{(1)}(\lambda) . c_{2}(\lambda)$ and $t_{(1)}(\lambda)$ are bounded for $\lambda \in \Omega$.

From (3.14) we conclude that a step-size sequence $h_{j}$ which equidistributes the local discretization error $\left(\left|l_{j+1 / 2}\right| \leqslant\right.$ const $\left.\varepsilon\right)$ and which fulfills $h_{j} t_{j+1 / 2}^{\alpha} \leqslant$ const implies

$$
\left|e_{i}\right| \leqslant \text { const } \varepsilon
$$

where const is independent of $\lambda \in \Omega$. We set $T=T(\varepsilon)$ as in (2.33) but substitute $\left(\lambda_{\min }-\delta\right)$ by $\lambda_{1}$.

(3.17) implies the bounds

$$
h_{i} \leqslant \bar{h}\left(\lambda_{1}, \varepsilon, t_{i}\right)= \begin{cases}c_{0} \sqrt{\varepsilon}, & 1 \leqslant t_{i} \leqslant \gamma, \gamma>0 \\ c_{1} \sqrt{\varepsilon} t_{i}^{-\alpha} \exp \left(\frac{\lambda_{1}}{2(\alpha+1)} t_{i}^{\alpha+1}\right), & \gamma \leqslant t_{i} \leqslant T(\varepsilon)\end{cases}
$$

for the mesh size $h_{i}$ at a point $t_{i}$, depending on $\varepsilon$ and $\lambda_{1}=\operatorname{Re} \lambda$. These bounds increase exponentially in $t$. Since $\bar{h}\left(\lambda_{1}, \varepsilon, T(\varepsilon)\right)=c_{1} T(\varepsilon)^{-\alpha} \sqrt{\varphi}$ holds, the condition $h_{i} t_{i+1 / 2}^{\alpha} \leqslant$ const is fulfilled and (3.18) holds on [1,T(E)]. The constants $c_{0}, c_{1}>0$ can be chosen arbitrarily; however, the constant in (3.18) is an increasing function of $c_{0}^{2}+c_{1}^{2}$.

We now compute the number of steps $N(\varepsilon)$ which is necessary for integration on the interval $[1, T(\varepsilon)]$ if $h_{i}=\bar{h}\left(\lambda_{1}, \varepsilon, t_{i}\right)$ is chosen. Therefore we set

$$
N(\varepsilon)=N_{1}(\varepsilon)+N_{2}(\varepsilon)
$$


where $N_{1}(\varepsilon)$ is the number of steps in $[1, \gamma]$ and $N_{2}(\varepsilon)$ is the number of steps in $[\gamma, T(\varepsilon)]$. Obviously

$$
N_{1}(\varepsilon) \leqslant \frac{\gamma-1}{c_{0} \sqrt{\varepsilon}}
$$

and

$$
\begin{aligned}
N_{2}(\varepsilon) & =\sum_{t_{i} \in[\gamma, T]} \frac{h_{i}}{h_{i+1}} \frac{h_{i+1}}{h_{i}} \\
& \leqslant \max _{t_{i} \in[\gamma, T]} \frac{h_{i+1}}{h_{i}} \int_{\gamma}^{T(\varepsilon)} \frac{d t}{\bar{h}(\lambda, \varepsilon, t)} \leqslant \frac{2}{\lambda_{1}} \exp \left(\frac{\lambda_{1}}{2}\left(\sqrt{\varphi}-\frac{\gamma^{\alpha+1}}{\alpha+1}\right)\right) \frac{1}{\sqrt{\varepsilon}}
\end{aligned}
$$

since $\max _{t_{i} \in[\gamma, T(\varepsilon)]} h_{i+1} / h_{i} \leqslant \exp \left(c_{1} \lambda_{1} \sqrt{\varphi} / 2\right)$ holds. From (3.21), (3.22) we conclude

$$
N(\varepsilon)=O\left(\frac{1}{\sqrt{\varepsilon}}\right)
$$

A constant step-size algorithm would need

$$
N_{\text {const }}(T, \varepsilon) \approx \frac{T(\varepsilon)}{\min \left(\sqrt{\varepsilon}, \sqrt[3]{\frac{\varepsilon}{T(\varepsilon)^{\alpha}}}\right)}
$$

steps because

$$
\max _{i}\left|\left(H_{-}\left(\lambda, t_{0}, h_{\text {const }}\right) l\right)_{i}\right| \leqslant \operatorname{const}\left(1+\frac{|\lambda|}{2} h T^{\alpha}\right) \max _{i}\left|l_{i+1 / 2}\right|, \quad t_{i+1} \leqslant T,
$$

holds, where $h_{\text {const }}=(h)_{i=0}^{N-1}$ is a sequence of constant step sizes.

Therefore the step-size sequence $\bar{h}$ given by (3.19) is very efficient and the reason is that no condition like $h_{\max } / h_{\min } \leqslant$ const is required. We remark that $\bar{h}$ equidistributes the local error.

The problem (3.1), (3.2) can be regarded as a model for the decaying solution components of boundary value problems on 'long' intervals, and now we look at the increasing components, which can be modelled by

$$
\begin{gathered}
z^{\prime}=\omega t^{\alpha} z+t^{\alpha} f(t), \quad \begin{array}{c}
1 \leqslant t \leqslant T, \alpha \geqslant 0, T=T(\varepsilon), \\
z(T)=\bar{z}
\end{array}
\end{gathered}
$$

where $\omega=\omega_{1}+i \omega_{2} \in \Omega$ and $\Omega$ is again a compact subset of $\{z \in \mathbf{C} \mid \operatorname{Re} z>0\}$. We again use the Box-scheme to approximate (3.25), (3.26)

$$
\frac{z_{i+1}-z_{i}}{h_{i}}=\frac{\omega}{2} t_{i+1 / 2}^{\alpha}\left(z_{i+1}+z_{i}\right)+t_{i+1 / 2}^{\alpha} f_{i+1 / 2}, \quad i \geqslant 0 ; \quad z_{N}=\bar{z}
$$

where

$$
\begin{aligned}
t_{0} & =1<t_{1}=t_{0}+h_{0}<\cdots<t_{N-1}=t_{N-2}+h_{n-2}<t_{N} \\
& =t_{N-1}+h_{N-1}=T(\varepsilon)
\end{aligned}
$$

holds. 
The solution of (3.27) is given by

$$
z_{i}=Y_{i, N-1}(\omega, h) \bar{z}+\left(H_{+}\left(\omega, t_{N}, h\right) f\right)_{i},
$$

where

$$
\left(H_{+}\left(\omega, t_{N}, h\right) f\right)_{i}=-\sum_{j=i}^{N-1} \frac{h_{j} t_{j+1 / 2}^{\alpha} f_{j+1 / 2}}{1+\frac{\omega}{2} h_{j} t_{j+1 / 2}^{\alpha}} Y_{i, j-1}(\omega, h), \quad i \leqslant N-1,
$$

and $\left(H_{+}\left(\omega, t_{N}, h\right) f\right)_{N}=0$.

Since the increasing components are scaled down by the asymptotic boundary conditions at $t=T$, we disregard the convergence of the $z_{i}$ to $z\left(t_{i}\right)$. Stability follows as in Lemma 3.2 .

LEMMA 3.3. Let $f=\left(f_{j+1 / 2}\right)_{j=1}^{N-1}$. Then for every sequence $h=\left(h_{j}\right)_{j=1}^{N-1}$

$$
\left|\left(H_{+}\left(\omega, t_{N}, h\right) f\right)_{i}\right| \leqslant \mathrm{const} \max _{j=i(1)(N-1)}\left[\left|f_{j+1 / 2}\right|\left(1+\frac{|\omega|}{2} h_{j} t_{j+1 / 2}^{\alpha}\right)\right]
$$

holds for $i \leqslant N$ uniformly for $\omega \in \Omega$.

Finally we prove

LEMMA 3.4. Assume that $t_{i} \leqslant t_{j} \leqslant T(\varepsilon)$ and that $h_{\kappa} \leqslant \bar{h}\left(\lambda_{1}, \varepsilon, t_{\kappa}\right), \lambda_{1}=\operatorname{Re} \lambda>0$. Then $\left|Y_{i, j-1}(\lambda, h)\right| \leqslant \exp \left(-c\left(t_{j}-t_{i}\right)\right)$, and $c=c(\lambda)$ is bounded on $\Omega$.

Proof. Let $z=z_{1}+i z_{2}, z_{1}>0$. Then

$$
\left|\frac{1-z}{1+z}\right|^{2} \leqslant 1-\frac{4 z_{1}}{|1+z|^{2}} \leqslant \exp \left(-4 \frac{z_{1}}{|1+z|^{2}}\right)
$$

holds. This estimate has been used in de Hoog and Weiss [6]. For $\lambda=\lambda_{1}+i \lambda_{2}$ we obtain

$$
\left|Y_{i, j-1}(\lambda, h)\right| \leqslant \exp \left(-2 \lambda_{1} \sum_{k=i}^{j-1} \frac{h_{k} t_{k+1 / 2}^{\alpha}}{\left|1+\frac{\lambda}{2} h_{k} t_{k+1 / 2}^{\alpha}\right|^{2}}\right) .
$$

Since $\left|1+(\lambda / 2) h_{k} t_{k+1 / 2}^{\alpha}\right|^{2} \leqslant c(\lambda)$ for $t_{k} \leqslant T(\varepsilon)$ holds, we get

$$
\begin{aligned}
\left|Y_{i, j-1}(\lambda, h)\right| & \leqslant \exp \left(-\frac{2 \lambda_{1}}{c(\lambda)} \sum_{k=i}^{j-1} h_{k} t_{k+1 / 2}^{\alpha}\right) \\
& \leqslant \exp \left(-c \sum_{k=i}^{j-1} h_{k}\right) \leqslant \exp \left(-c\left(t_{j}-t_{i}\right)\right) .
\end{aligned}
$$

4. Linear Boundary Value Problems. We consider

$$
\begin{gathered}
x^{\prime}=t^{\alpha} A x+t^{\alpha} f(t), \quad 1 \leqslant t \leqslant T, \alpha \geqslant 0, \\
B x(1)=\beta, \\
S(T) x(T)=\sigma(T),
\end{gathered}
$$

where $A$ fulfills (2.4), (2.5), $B$ is an $r_{-} \times n$-matrix, $\beta \in R^{r-}$ and $f$ fulfills (2.13) with $\theta>\lambda_{\text {min }}$, the $r_{+} \times n$-matrix $S(T)$ fulfills (2.29), (2.30) and $T=T(\varepsilon)$ as in (2.23). This simple case is considered as a model for problems where $A$ depends on $t$. 
The Box-scheme has the form

$$
\begin{aligned}
\frac{x_{i+1}-x_{i}}{h_{i}}=\frac{A}{2} t_{i+1 / 2}^{\alpha}\left(x_{i+1}+x_{i}\right) & +t_{i+1 / 2}^{\alpha} f_{i+1 / 2}, \quad i=0(1)(N-1), \\
B x_{0} & =\beta, \\
S(T) x_{N} & =\sigma(T),
\end{aligned}
$$

where the partition $\Delta=\left\{t_{0}, t_{1}, \ldots, t_{N-1}, t_{N}\right\}$ fulfills (3.4)(a) and $h=\left(h_{i}\right)_{i=0}^{N-1}, h_{i}>0$. $A$ fulfills (2.4). We employ the transformation

$$
x_{i}=E u_{i}
$$

and get

$$
\begin{aligned}
\frac{u_{i+1}-u_{i}}{h_{i}}=\frac{J}{2} t_{i+1 / 2}^{\alpha}\left(u_{i+1}+u_{i}\right)+ & t_{i+1 / 2}^{\alpha} E^{-1} f_{i+1 / 2}, \quad i=0(1)(N-1), \\
B E u_{0} & =\beta, \\
S(T) E u_{N} & =\sigma(T) .
\end{aligned}
$$

We want to derive an existence and stability theorem for (4.8), (4.9), (4.10). As de Hoog and Weiss [7] did for the continuous case we split $u_{i}$ into
(a) $\left.u_{i}=\left(\begin{array}{c}u_{i}^{+} \\ u_{i}^{-}\end{array}\right)\right\} r_{+}$
(b) $\left.E^{-1} f_{i+1 / 2}=\left(\begin{array}{l}\left(E^{-1} f_{i+1 / 2}\right)^{+} \\ \left(E^{-1} f_{i+1 / 2}\right)^{-}\end{array}\right)\right\} r_{+}$,

and get employing (3.6) for $u_{i}^{-}$and (3.29) for $u_{i}^{+}$

$$
\begin{aligned}
u_{i}= & {\left[\begin{array}{c}
Y_{i, N-1}^{r_{+}}\left(J^{+}, h\right) \\
0
\end{array}\right] \xi_{+}+\left[\begin{array}{c}
0 \\
Y_{0, \bar{i}-1}^{r}\left(-J^{-}, h\right) e^{J^{-}}
\end{array}\right] \xi_{-} } \\
& +\left(H\left(J, t_{0}, t_{N}, h\right) E^{-1} f\right)_{i},
\end{aligned}
$$

where for any $k \times k$ matrix $P$ whose eigenvalues have positive real part

$$
\begin{gathered}
Y_{n, m}^{k}(P, h)=\prod_{j=n}^{m}\left(I_{k}+\frac{P}{2} h_{j} t_{j+1 / 2}^{\alpha}\right)^{-1}\left(I_{k}-\frac{P}{2} h_{j} t_{j+1 / 2}^{\alpha}\right), \quad m \geqslant n, \\
Y_{n+1, n}(P, h)=I, \quad n \geqslant-1,
\end{gathered}
$$

holds and the operator $H$ is defined as

$$
\text { (4.14)(a) } \quad\left(H\left(J, t_{0}, t_{N}, h\right) z\right)_{i}=\left(\begin{array}{c}
\left(H_{+}\left(J^{+}, t_{N}, h\right) z^{+}\right)_{i} \\
\left(H_{-}\left(-J^{-}, t_{0}, h\right) z^{-}\right)_{i}
\end{array}\right), \quad z=\left(\begin{array}{c}
z^{+} \\
z^{-}
\end{array}\right)
$$

and

(4.14)(b) $\quad\left(H_{+}\left(J^{+}, t_{N}, h\right) z^{+}\right)_{i}$

$$
=-\sum_{j=i}^{N-1} h_{j} Y_{i, j-1}^{r_{+}}\left(J^{+}, h\right)\left(I+\frac{J^{+}}{2} h_{j} t_{j+1 / 2}^{\alpha}\right)^{-1} t_{j+1 / 2}^{\alpha} z_{j+1 / 2}^{+},
$$

$(4.14)(\mathrm{c}) \quad\left(H_{-}\left(-J^{-}, t_{I}, h\right) z^{-}\right)_{i}$

$$
=\sum_{j=I}^{i-1} h_{j} Y_{j+1, i-1}^{r}\left(-J^{-}, h\right)\left(I-\frac{J^{-}}{2} h_{j} t_{j+1 / 2}^{\alpha}\right)^{-1} t_{j+1 / 2}^{\alpha} z_{j+1 / 2}^{-},
$$


where $z^{+}=\left(z_{i+1 / 2}^{+}\right)_{i=0}^{N-1}, z^{-}=\left(z_{i+1 / 2}^{-}\right)_{i=0}^{N-1}$ and $z_{i+1 / 2}^{+} \in \mathbf{C}^{r_{+}}, z_{i+1 / 2}^{-} \in \mathbf{C}^{r_{-}}$has been set. Here $\left(H_{+}\left(J^{+}, t_{N}, h\right) z^{+}\right)_{N}=0$ and $\left(H_{-}\left(-J^{-}, t_{I}, h\right) z^{-}\right)_{I}=0$ hold. These definitions make sense because $\left(I+\tau J^{+}\right)^{-1},\left(I-\tau J^{-}\right)^{-1}$ exist for $\tau \geqslant 0$.

In order to get bounds for the defined difference operators, we use the following representation of a matrix function

$$
\phi(P)=\frac{1}{2 \pi i} \int_{\Gamma_{P}} \phi(\lambda)(\lambda I-P)^{-1} d \lambda,
$$

where the contour $\Gamma_{P}$ encloses all eigenvalues of $P$. $\phi$ is assumed to be analytic. (4.15) has been used by de Hoog and Weiss [6] in a similar context.

If all eigenvalues of $P$ have positive real parts, we get

$$
Y_{n, m}^{k}(P, h)=\frac{1}{2 \pi i} \int_{\Gamma_{P}} Y_{n, m}(\lambda, h)\left(\lambda I_{k}-P\right)^{-1} d \lambda,
$$

where $Y_{n, m}$ is defined in (3.4) and

$$
\left(H\left(J, t_{0}, t_{N}, h\right) z\right)_{i}=\frac{1}{2 \pi i}\left(\begin{array}{l}
\int_{\Gamma_{+}}\left(H_{+}\left(\omega, t_{N}, h\right)\left(\omega I-J^{+}\right)^{-1} z\right)_{i} d \omega \\
\int_{\Gamma_{-}}\left(H_{-}\left(-\lambda, t_{0}, h\right)\left(\lambda I-J^{-}\right)^{-1} z^{-}\right)_{i} d \lambda
\end{array}\right),
$$

where $\Gamma_{+} \subset\{z \in \mathbf{C} \mid \operatorname{Re} z>0\}, \Gamma_{-} \subset\{z \in \mathbf{C} \mid \operatorname{Re} z<0\}$ holds. Since

$$
\max _{\omega \in \Gamma_{+}}\left\|\left(\omega I-J^{+}\right)^{-1}\right\|, \max _{\lambda \in \Gamma_{-}}\left\|\left(\lambda I-J^{-}\right)^{-1}\right\| \leqslant \text { const, }
$$

the estimates given in Section 3 can be used because they were formulated uniformly for $-\lambda, \omega$ in compact subsets of the left half plane.

By evaluating (4.12) at the boundaries $(i=1, i=N)$ we get the block system

$$
\begin{gathered}
{\left[\begin{array}{cc}
B E\left[\begin{array}{c}
Y_{0, N-1}^{r_{+}}\left(J^{+}, h\right) \\
0
\end{array}\right] & B E\left[\begin{array}{c}
0 \\
e^{J^{-}}
\end{array}\right] \\
S(T) E\left[\begin{array}{l}
I \\
0
\end{array}\right] & S(T) E\left[\begin{array}{c}
0 \\
Y_{0, N-1}^{r_{-}}\left(-J^{-}, h\right)
\end{array}\right]
\end{array}\right]\left(\begin{array}{l}
\xi_{+} \\
\xi_{-}
\end{array}\right)} \\
=\left(\begin{array}{c}
\beta-B E\left(H\left(J, t_{0}, t_{N}, h\right) E^{-1} f\right)_{0} \\
\sigma(T)-S(T) E\left(H\left(J, t_{0}, t_{N}, h\right) E^{-1} f\right)_{N}
\end{array}\right)
\end{gathered}
$$

We assume that (2.1), (2.2), (2.3) with $A(T) \equiv A$ has a unique solution for all $f \in C([1, \infty]), \beta \in R^{r}$. Therefore (2.15) holds and implies that

$$
B E\left[\begin{array}{c}
0 \\
e^{J^{-}}
\end{array}\right]
$$

is nonsingular. From (2.30) we conclude that $S(T) E\left[\begin{array}{l}I \\ 0\end{array}\right]$ has a bounded inverse. From (4.16) we conclude that

$$
\left\|Y_{0, N-1}^{r_{+}}\left(J^{+}, h\right)\right\| \leqslant \mathrm{const} \max _{\omega \in \Gamma_{+}}\left|Y_{0, N-1}(\omega, h)\right| \leqslant \mathrm{const}
$$

and

$$
\left\|Y_{0, N-1}^{r^{\prime}-1}\left(-J^{-}, h\right)\right\| \leqslant \text { const } \max _{\lambda \in \Gamma_{-}}\left|Y_{0, N-1}(-\lambda, h)\right| .
$$


Now let $\bar{\lambda}$ be the eigenvalue of $J^{-}$which is nearest to the imaginary axis, such that $\operatorname{Re} \bar{\lambda}=-\lambda_{\min }$, and take $\Gamma_{-}$such that

(4.22) $\operatorname{dist}\left(\Gamma_{-}, \bar{\lambda}\right)=\delta$ and $\operatorname{dist}\left(\Gamma_{-},\{z \in C \mid \operatorname{Re} z=0\}\right)=\lambda_{\min }-\delta$

holds. We now choose $h=\left(h_{i}\right)_{i=0}^{N-1}$ such that $h_{i} \leqslant \bar{h}\left(\lambda_{\min }-\delta, \varepsilon, t_{i}\right)$ defined in (3.19).

Because of Lemma 3.4

$$
\max _{\lambda \in \Gamma_{-}}\left|Y_{0, N-1}(-\lambda, h)\right|=o(1), \quad \varepsilon \rightarrow 0,
$$

holds. Therefore, for $\varepsilon$ sufficiently small $\left\|Y_{0, N_{-1}}^{r_{-1}}\left(-J^{-}, h\right)\right\|$ can be made sufficiently small such that the block system (4.19) has a unique solution $\xi_{+}, \xi_{-}$.

(4.17) and the Lemmas 3.2, 3.3 yield

$$
\max _{i=0(1)(N-1)}\left\|\left(H(J, 1, T(\varepsilon), h) E^{-1} f\right)_{i}\right\| \leqslant \text { const } \max _{i=0(1)(N-1)}\left\|f_{i+1 / 2}\right\|,
$$

and the stability estimate

$$
\max _{i=0(1) N}\left\|x_{i}\right\| \leqslant \operatorname{const}\left(\|\beta\|+\|\sigma(T(\varepsilon))\|+\max _{i=0(1)(N-1)}\left\|f_{i+1 / 2}\right\|\right)
$$

follows.

The local discretization error $t_{i+1 / 2}^{\alpha} l_{i+1 / 2}$ is again defined as

$$
\begin{gathered}
t_{i+1 / 2}^{\alpha} l_{i+1 / 2}=\frac{x\left(t_{i+1}\right)-x\left(t_{i}\right)}{h_{i}}-\frac{A}{2} t_{i+1 / 2}^{\alpha}\left(x\left(t_{i+1}\right)+x\left(t_{i}\right)\right) \\
-t_{i+1 / 2}^{\alpha} f_{i+1 / 2}, \quad i=0(1)(N-1),
\end{gathered}
$$

such that the global discretization error

$$
e_{i}=x\left(t_{i}\right)-x_{i}
$$

fulfills the discrete boundary value problem

$$
\begin{gathered}
\frac{e_{i+1}-e_{i}}{h_{i}}=\frac{A}{2} t_{i+1 / 2}^{\alpha}\left(e_{i+1}+e_{i}\right)+t_{i+1 / 2}^{\alpha} l_{i+1 / 2}, \quad i=0(1)(N-1), \\
B e_{0}=0 \\
S(T) e_{N}=0 .
\end{gathered}
$$

From (4.26) we obtain

$$
\max _{i=0(1) N}\left\|e_{i}\right\| \leqslant \mathrm{const} \max _{i=0(1) N}\left\|l_{i+1 / 2}\right\|
$$

where $l=\left(l_{i+1 / 2}\right)_{i=0}^{N-1}$ has been set.

As in Section 3 we obtain

$$
\left\|l_{i+1 / 2}\right\| \leqslant \text { const } h_{i}^{2}\left[\frac{1}{t_{i+1 / 2}^{\alpha}}\left\|x^{\prime \prime \prime}\right\|_{\left[t_{i}, t_{i+1}\right]}+\left\|x^{\prime \prime}\right\|_{\left[t_{i}, t_{i+1}\right]}\right],
$$

assuming that (2.13) holds with $F, F^{\prime}, F^{\prime \prime} \in C([1, \infty)) \cap L_{\infty}([1, \infty])$. Now let $\sigma(T)$ $=0$. Since

$$
\|x(t)\| \leqslant\|y(t)\|+\|x(t)-y(t)\| \leqslant\|y(t)\|+\text { const }\|S(T) y(T)\|
$$


holds, where $y$ is the (unique) solution of the 'infinite' problem (2.1), (2.2), (2.3) with $A(T) \equiv A$, we get

$$
\begin{array}{r}
\|x(t)\| \leqslant \text { const }\left(\left(\sum_{\kappa=0}^{2}\left\|F^{(\kappa)}\right\|_{[1, \infty]}+\|\beta\|\right) \exp \left(-\frac{\left(\lambda_{\min }-\delta\right)}{\alpha+1} t^{\alpha+1}\right)\right. \\
+\|S(T) y(T)\|)
\end{array}
$$

This follows from (2.14), (2.28). By using the differential equation (4.1), we derive

$$
\left\|l_{i+1 / 2}\right\| \leqslant \text { const } h_{i}^{2}\left(t_{i}^{2 \alpha} \exp \left(-\frac{\left(\lambda_{\min }-\delta\right)}{\alpha+1} t_{i}^{\alpha+1}\right)\right)+O(\varepsilon)
$$

Since $h_{i} \leqslant \bar{h}\left(\lambda_{\min }-\delta, \varepsilon, t_{i}\right)$, we obtain

$$
\max _{i=0(1) N}\left\|e_{i}\right\|=O(\varepsilon)
$$

The estimate (2.14) for $y(T)$ gives a bound for the total error

$$
\text { (4.36) } \max _{i=O(1) N}\left\|y\left(t_{i}\right)-x_{i}\right\| \leqslant \text { const }\left(\varepsilon+\exp \left(-\frac{\left(\lambda_{\min }-\delta\right)}{\alpha+1} T(\varepsilon)^{\alpha+1}\right)\right)=O(\varepsilon) \text {. }
$$

Again $y$ solves the 'infinite' problem and $x_{i}$ are the solutions of the Box-scheme for the 'finite' problem on $[1, T(\varepsilon)]$.

We briefly investigate linear problems where the coefficient matrix $A$ depends on $t$.

The Box-scheme for the approximating 'finite' problem is

$$
\begin{gathered}
\frac{x_{i+1}-x_{i}}{h_{i}}=\frac{1}{2} t_{t+1 / 2}^{\alpha} A\left(t_{i+1 / 2}\right)\left(x_{i+1}+x_{i}\right)+t_{i+1 / 2}^{\alpha} f_{i+1 / 2}, \\
B x_{0}=\beta, \\
S(T) x_{N}=\sigma(T), \quad T=T(\varepsilon)=t_{N} .
\end{gathered}
$$

For the $n \times n$-matrix, $A \in C([1, \infty])$ is assumed to hold and $A(\infty)=\lim _{t \rightarrow \infty} A(t)$ has the Jordan form $J$ obtained by

$$
A(\infty)=E J E^{-1},
$$

and $J$ has the block structure (2.5). Again we set

$$
x_{i}=E u_{i}
$$

and define

$$
G(t)=E^{-1} A(t) E-J ; \quad G(t) \rightarrow 0, t \rightarrow \infty .
$$

We use a perturbation approach for the derivation of an existence and stability theorem and rewrite (4.37), (4.38), (4.39)

$$
\begin{aligned}
\frac{u_{i+1}-u_{i}}{h_{i}}=\frac{J}{2} t_{i+1 / 2}^{\alpha}\left(u_{i+1}+u_{i}\right)+\frac{1}{2} t_{i+1 / 2}^{\alpha} G\left(t_{i+1 / 2}\right)\left(u_{i+1}+u_{i}\right) & \\
+t_{i+1 / 2}^{\alpha} E^{-1} f_{i+1 / 2}, \quad i=0(1)(N-1) & \\
B E u_{0} & =\beta \\
S(T) E u_{N} & =\sigma(T)
\end{aligned}
$$


The general solution of (4.43) can be written as

$$
\begin{gathered}
u_{i}=\left[\begin{array}{c}
Y_{i, N-1}^{r_{+}}\left(J^{+}, h\right) \\
0
\end{array}\right] \xi_{+}+\left[\begin{array}{c}
0 \\
Y^{r_{-}}\left(-J^{-}, h\right) e^{J^{-}}
\end{array}\right] \xi_{-} \\
+\left(H\left(J, t_{I}, t_{N}, h\right) \tilde{G} u\right)_{i}+\left(H\left(J, t_{I}, t_{N}, h\right) E^{-1} f\right)_{i} \cdot \\
(\tilde{G} u)_{i+1 / 2}=\frac{1}{2} G\left(t_{i+1 / 2}\right)\left(u_{i+1}+u_{i}\right), \quad \tilde{G} u=\left((\tilde{G} u)_{i+1 / 2}\right)_{i=I}^{N-1}
\end{gathered}
$$

has been set. $\xi_{+} \in C^{r_{+}}$and $\xi_{-} \in C^{r}$ - hold.

From (4.17) and the Lemmas 3.2 and 3.3 we get

$$
\max _{i=I(1) N}\left\|\left(H\left(J, t_{I}, t_{N}, h\right) \tilde{G} u\right)_{i}\right\| \leqslant \text { const }\|G\|_{\left[t_{t}, t_{N}\right]} \max _{i=I(1) N}\left\|u_{i}\right\|,
$$

where $h_{i} \leqslant h\left(\lambda_{\min }-\delta, \varepsilon, t_{i}\right)$ is assumed to hold. $\lambda_{\min }>0$ is the modulus of the real part of that eigenvalue of $A(\infty)$ which is closest to the imaginary axis of all eigenvalues of $A(\infty)$ with negative real part.

We define the operator

$$
\hat{H}\left(h, t_{I}, t_{N}\right): C^{n(N-1)} \rightarrow C^{n(N-I+1)}
$$

such that, for $\bar{x} \in \mathbf{C}^{n(N-1)}, \bar{x}=\left(\bar{x}_{i+1 / 2}\right)_{i=I}^{N-1}$,

$$
\hat{H}\left(h, t_{I}, t_{N}\right) \bar{x}=\left(\begin{array}{c}
\left(H\left(J, t_{I}, t_{N}, h\right) \bar{x}\right)_{I} \\
\vdots \\
\left(H\left(J, t_{I}, t_{N}, h\right) \bar{x}\right)_{N}
\end{array}\right)
$$

holds. From (4.42), (4.48) we get

$$
\left\|\hat{H}\left(h, t_{I}, t_{N}\right) \tilde{G}\right\| \leqslant \mathrm{const}\|G\|_{\left[t_{I}, t_{N}\right]},
$$

where $\|\cdot\|$ denotes the max-norm for vectors in the respective $\mathbf{C}^{j}$ or the associated matrix norm. Therefore the operator

$$
I-\hat{H}\left(h, t_{I}, t_{N}\right) \tilde{G}: \mathbf{C}^{n(N-I+1)} \rightarrow \mathrm{C}^{n(N-I+1)}
$$

is invertible for $t_{I}<t_{N}$ sufficiently large.

The existence theorem for (4.37), (4.38), (4.39) follows by proceeding as de Hoog and Weiss [8] did for the continuous 'finite' problem (2.23), (2.24), (2.25). The stability estimate (4.25) holds for variable coefficient problems too.

TheOREM 4.1. Assume that $A \in C([1, \infty]) ; A^{\prime}, A^{\prime \prime} \in C([1, \infty)) \cap L_{\infty}([1, \infty])$ and that $f$ fulfills $(2.13)$ with $F, F^{\prime}, F^{\prime} \in C([1, \infty)) \cap L_{\infty}([1, \infty])$. Let, for some $\varepsilon$ sufficiently small, $T=T(\varepsilon)=t_{N}$ as in (2.23), and assume that

$$
\begin{array}{cl}
h_{i} \leqslant c_{0} \cdot \sqrt{\varepsilon}, \quad t_{i} \leqslant \gamma, & c_{0}>0, \\
h_{i} \leqslant \sqrt{\varepsilon} t_{i}^{-\alpha} \exp \left(\frac{\lambda_{\min }-\delta}{\alpha+1} t_{i}^{\alpha+1}\right), & \gamma \leqslant t_{i}<T(\varepsilon),
\end{array}
$$

holds for some fixed $\gamma$. Then if the matrix (2.18) is nonsingular, the Box-scheme (4.37), (4.38), (4.39) is uniquely soluble and

$$
\max _{i=0(1) N}\left\|x_{i}-y\left(t_{i}\right)\right\|=O(\varepsilon)
$$

holds for $\sigma(T) \equiv 0$. 
For the remainder of this section we assume that equality holds in (4.53), (4.54). Then the number of steps $N=N(\varepsilon)$ fulfills

$$
N(\varepsilon) \sim c \cdot \frac{1}{\sqrt{\varepsilon}}, \quad \varepsilon \rightarrow 0 .
$$

The condition number of a nonsingular matrix $A$ is defined by

$$
x(A)=\|A\|\left\|A^{-1}\right\| .
$$

The condition number of the difference operator $L_{h}$ (given by (4.37), (4.38), (4.39), where both sides of (4.37) are divided by $t_{i+1 / 2}^{\alpha}$ ), fulfills the estimate

$$
x\left(L_{h}\right) \leqslant \text { const } \frac{1}{\sqrt{\varepsilon}} \sim \text { const } N(\varepsilon)
$$

since

$$
\left\|L_{h}\right\| \leqslant \max \left(\|B\|,\|S(T(\varepsilon))\|, \max _{i=0(1)(N-1)} \frac{2}{t_{i+1 / 2}^{\alpha} h_{i}}+\|A\|_{[1, \infty]}\right)
$$

and because the stability estimate (4.25) implies $\left\|L_{h}^{-1}\right\| \leqslant$ const. (4.58) is a very moderate condition number, and therefore (4.37), (4.38), (4.39) can be safely solved by Gaussian elimination with partial pivoting using for example the code SOLVEBLOCK (de Boor and Weiss [5]).

5. Nonlinear Problems. We consider the 'infinite' problem

$$
\begin{gathered}
y^{\prime}=t^{\alpha} f(t, y), \quad 1 \leqslant t<\infty, \alpha \geqslant 0, \\
b(y(1))=0, \\
y \in C([1, \infty]),
\end{gathered}
$$

and the Box-scheme

$$
\begin{gathered}
\frac{x_{i+1}-x_{i}}{h_{i}}=t_{i+1 / 2}^{\alpha} f\left(t_{i+1 / 2}, \frac{1}{2}\left(x_{i+1}+x_{i}\right)\right), \quad i=0(1)(N-1), \\
b\left(x_{0}\right)=0, \\
S(T) x_{N}=S(T) y^{*}(\infty),
\end{gathered}
$$

where $T=t_{N}$ holds. The asymptotic boundary condition $S(T)$ is set up as described in Section 2.

As mentioned in Section 2,

$$
f\left(\infty, y^{*}(\infty)\right)=0
$$

has to hold. We now assume that $y^{*}(\infty)$ is an isolated zero and that $f_{y}\left(\infty, y^{*}(\infty)\right)$ has the Jordan form $J$ obtained by

$$
f_{y}\left(\infty, y^{*}(\infty)\right)=E J E^{-1}
$$

where $J$ fulfills (2.5). Moreover, we assume

$$
f \in C^{2}\left(C_{x}\left(1, y^{*}(\infty)\right)\right) ; f\left(t, y^{*}(\infty)\right)=O\left(e^{-(\theta /(\alpha+1)) t^{\alpha+1}}\right), \quad \theta>\lambda_{\min },
$$
$b: R^{n} \rightarrow R^{r_{-}} ; b, b_{y}$ are locally Lipschitz continuous in $R^{n}$, 
and that the problem (5.1), (5.2), (5.3) has an isolated solution $y^{*}(t) \rightarrow y^{*}(\infty)$ as $t \rightarrow \infty$. The isolatedness means that the linearized problem

$$
z^{\prime}=f_{y}\left(t, y^{*}(t)\right) z, \quad b_{y}\left(y^{*}(1)\right) z(1)=0, \quad z \in C([1, \infty])
$$

has only the trivial solution $z \equiv 0$. We set $T=T(\varepsilon)$ as defined in (2.33) and conclude from de Hoog and Weiss [7] that the approximating problems

$$
\begin{gathered}
x^{\prime}=t^{\alpha} f(t, x), \\
b(x(1))=0, \\
S(T) x(T)=S(T) y^{*}(\infty),
\end{gathered}
$$

with (2.29), (2.30), are locally (around $\left.y^{*}(t)\right)$ uniquely soluble for $T(\varepsilon)$ sufficiently large and

$$
\|x-y\|_{[1, T]} \leqslant \text { const }\left\|S(T)\left(y(T)-y^{*}(\infty)\right)\right\| \leqslant \text { const }\|S(T)\| \varepsilon
$$

holds. Possible choices for $S(T)$ are discussed in Lentini and Keller [10].

We apply the nonlinear stability theory given in Keller [9] with $\varepsilon$ as a grid parameter. The result then follows from the stability estimate (4.25) for linear problems, and we merely state it in

THEOREM 5.1. Under the given assumption the Box-scheme has (for $\varepsilon$ sufficiently small) a locally unique solution which converges to the locally unique solution of (5.4), (5.5), (5.6) if a step-size sequence $h_{i}$ fulfilling

$$
\begin{gathered}
h_{i} \leqslant c_{0} \sqrt{\varepsilon}, \quad t_{i} \leqslant \gamma, \\
h_{i} \leqslant \sqrt{\varepsilon} t_{i}^{-\alpha} \exp \left(\frac{\lambda_{\min }-\delta}{2(\alpha+1)} t_{i}^{\alpha+1}\right), \quad \gamma<t_{i} \leqslant T(\varepsilon),
\end{gathered}
$$

is chosen. The estimate

$$
\max _{i=0(1) N}\left\|y\left(t_{i}\right)-x_{i}\right\|=O(\varepsilon), \quad \varepsilon \rightarrow 0,
$$

holds. The Newton procedure for (5.4), (5.5), (5.6) is quadratically convergent for starting values in a sphere

$$
K=\left\{x \in C^{n(N+1)} \mid\left\|x-\left[\begin{array}{c}
y\left(t_{0}\right) \\
\vdots \\
y\left(t_{N}\right)
\end{array}\right]\right\| \leqslant \xi\right\},
$$

where $\xi>0$ is independent of $\varepsilon$.

The mesh-size sequence (5.16) can be employed on the whole interval [1, T( $\varepsilon)]$ (since $h\left(\lambda_{\min }-\delta, \varepsilon, t_{i}\right) \leqslant \sqrt{\varepsilon} \gamma^{-\alpha} \exp \left(\left(\left(\lambda_{\min }-\delta\right) / 2(\alpha+1)\right) \gamma^{\alpha+1}\right)$ holds on $\left.[1, \gamma]\right)$ however, there might be more efficient choices on that interval where $y$ is not close to $y^{*}(\infty)$.

6. Higher Order Methods. It is intriguing to use symmetric, $A$-stable (higher order) collocation methods (see de Boor and Swartz [4], Russell [17] and Weiss [20]) for the solution of (5.11), (5.12), (5.13). We define

$$
t_{i j}=t_{i}+\rho_{j} h_{i}, \quad 0<\rho_{1}<\rho_{2}<\cdots<\rho_{k-1}<\rho_{k}<1,
$$


and require that the $\rho_{i}$ are symmetric about $\frac{1}{2}$. We do not include $\rho_{i}=0, \rho_{k}=1$ (for example Lobatto points) in this discussion. However, the results carry over to this case if the mesh-size sequence $h_{j}$ fulfills $h_{j} t_{j+1}^{\alpha} \leqslant$ const as $T=T(\varepsilon) \rightarrow \infty$ where const is sufficiently small but independent of $\varepsilon$. If mesh-size sequences which grow faster are admitted and if the endpoints 0,1 are collocation points, then instabilities can occur in the exponentially increasing components in the case $\alpha>0$.

This is illustrated by the homogeneous problem

$$
z^{\prime}=2 t z, \quad z(T)=\bar{z} .
$$

The trapezoidal rule has the solutions

$$
z_{i}=\prod_{j=i}^{N-1} \frac{1-h_{j} t_{j+1}}{1+h_{j} t_{j}} \bar{z}
$$

If $h_{j}>\sqrt{1+\rho}$ for $\rho>1, i \leqslant j \leqslant N-1$, then $\left|\left(1-h_{j} t_{j+1}\right) /\left(1+h_{j} t_{j}\right)\right|>\rho>1$ holds. Keeping $t=t_{i}$ (and the mesh on $\left[1, t_{i}\right]$ ) fixed implies $\left|z_{i}\right|>(\rho)^{N-i}|\bar{z}| \rightarrow \infty$ as $N \rightarrow \infty(T \rightarrow \infty)$. This exponential instability does not occur when using the midpoint rule.

We write the $k$-stage collocation method for (5.11), (5.12), (5.13) as a system of difference equations

$$
\begin{gathered}
\frac{x_{i j}-x_{i}}{h_{i}}=\sum_{l=1}^{k} \hat{a}_{j l} t_{i l}^{\alpha} f\left(t_{i l}, x_{i l}\right), \quad i=0(1)(N-1), j=1(1) k, \\
\frac{x_{i+1}-x_{i}}{h_{i}}=\sum_{l=1}^{k} \hat{b}_{l} t_{i l}^{\alpha} f\left(t_{i l}, x_{i l}\right), \quad i=0(1)(N-1), \\
b\left(x_{0}\right)=0, \\
S(T) x_{N}=S(T) y^{*}(\infty), \quad T=T(\varepsilon),
\end{gathered}
$$

where the $x_{i j}$ are the approximations to $x\left(t_{i j}\right)$ (resp. to $\left.y\left(t_{i j}\right)\right)$. From Ascher and Weiss [2] we get

$$
\hat{a}_{j l}=\varphi_{l}\left(\rho_{j}\right), \quad \hat{b}_{l}=\varphi_{l}(1),
$$

where $\varphi_{l}$ is the interpolating polynomial (of degree $k$ ) which fulfills

$$
\varphi_{l}(0)=0 ; \quad \varphi_{l}^{\prime}\left(\rho_{j}\right)=\delta_{l j}, \quad j=1(1) k .
$$

We set $\hat{A}=\left(\hat{a}_{j l}\right)_{j=1(1) k ; l=1(1) k}, \hat{b}=\left(\hat{b}_{1}, \ldots, \hat{b}_{k}\right)^{\prime}$, where the superscript " "'” denotes transposition. The eigenvalues of $A$ have positive real parts. In particular the Gauss-schemes belong to this class ( $\rho_{i}$ are the zeros of Gauss-Legendre polynomials) and the Box-scheme is the one-stage Gauss-scheme (with $\rho_{1}=\frac{1}{2}$ ).

At first we investigate the stability of

$$
\begin{gathered}
\frac{y_{i j}-y_{i}}{h_{i}}=-\lambda t_{i+1 / 2}^{\alpha} \sum_{l=1}^{k} \hat{a}_{j l} y_{i l}+t_{i+1 / 2}^{\alpha} f_{i j}, \quad y_{0}=\bar{y}, \\
\frac{y_{i+1}-y_{i}}{h_{i}}=-\lambda t_{i+1 / 2}^{\alpha} \sum_{l=1}^{k} \hat{b}_{l} y_{i l}+t_{i+1 / 2}^{\alpha} f_{i},
\end{gathered}
$$


where $\operatorname{Re} \lambda>0 ; y_{i j}, y_{i}, f_{i j}, f_{i} \in \mathbf{C}$ holds. Setting $\overrightarrow{1}=(1, \ldots, 1)^{\prime}, \vec{y}_{i}=\left(y_{i 1}, \ldots, y_{i k}\right)^{\prime}$, $\overrightarrow{f_{i}}=\left(f_{i 1}, \ldots, f_{i k}\right)^{\prime}$, we obtain

(6.10) $\quad \vec{y}_{i}=\left(I+\lambda h_{i} t_{i+1 / 2}^{\alpha} \hat{A}\right)^{-1} \overrightarrow{1} y_{i}+h_{i} t_{i+1 / 2}^{\alpha}\left(I+\lambda h_{i} t_{i+1 / 2}^{\alpha} \hat{A}\right)^{-1} \vec{f}_{i}$,

(6.11)(a) $\quad y_{i+1}=\left(1-\lambda h_{i} t_{i+1 / 2}^{\alpha} \hat{b^{\prime}}\left(I+\lambda h_{i} t_{i+1 / 2}^{\alpha} \hat{A}\right)^{-1} \overrightarrow{1}\right) y_{i}$

$$
-\lambda h_{i}^{2} t_{i+1 / 2}^{2 \alpha} \hat{b}^{\prime}\left(I+\lambda h_{i} t_{i+1 / 2}^{\alpha} \hat{A}\right)^{-1} \vec{f}_{i}+h_{i} t_{i+1 / 2}^{\alpha} f_{i} \text {. }
$$

The function

(6.11)(b)

$$
\gamma(z)=1-z \hat{b}^{\prime}(I+z \hat{A})^{-1} \overrightarrow{1}
$$

is the growth function of the collocation scheme and fulfills

$$
|\gamma(z)| \leqslant 1, \quad \operatorname{Re} z \geqslant 0 \text { (A-stability) }
$$

$$
|\gamma(z)|=1 \Rightarrow \operatorname{Re} z=0 \text { or } z=\infty, \quad(\operatorname{Re} z \geqslant 0),
$$

$$
\lim _{z \rightarrow \infty}(1-|\gamma(z)|) /|z|>0 \quad \text { for } \operatorname{Re} z>0,\left|\frac{\operatorname{Im} z}{\operatorname{Re} z}\right| \leqslant c,
$$

$$
\gamma(z)=e^{z}+O\left(z^{k+1}\right), \quad z \rightarrow 0, \operatorname{Re} z \geqslant 0,
$$

(see Ascher and Weiss [2]).

From (6.11) we get

$$
y_{i}=\prod_{m=0}^{i-1} \gamma\left(z_{m}\right) \bar{y}+\frac{1}{\lambda} \sum_{j=0}^{i-1} z_{j}\left(-z_{j} \hat{b}^{\prime}\left(I+z_{j} \hat{A}\right)^{-1} \vec{f}_{j}+f_{j}\right) \prod_{m=j+1}^{i-1} \gamma\left(z_{m}\right),
$$

where $z_{j}=-\lambda h_{j} t_{j+1 / 2}^{\alpha}$ has been set. A simple calculation shows that

$$
\begin{aligned}
& |z|\left(|z|\left|\hat{b}^{\prime}\right|\left\|(I+z \hat{A})^{-1}\right\|\left\|\overrightarrow{f_{j}}\right\|+\left|f_{j}\right|\right) \\
& \quad \leqslant|z| \max \left(|z|\left\|\overrightarrow{f_{j}}\right\|+\left|f_{j}\right|\right), \quad \operatorname{Re} z \geqslant 0,|\operatorname{Im} z / \operatorname{Re} z| \leqslant c
\end{aligned}
$$

Using (6.12), (6.13), (6.14), we obtain

$$
\frac{|z|}{|1+z|^{2}} \leqslant \text { const }(1-|\gamma(z)|), \quad \operatorname{Re} z>0,\left|\frac{\operatorname{Im} z}{\operatorname{Re} z}\right|<c .
$$

Proceeding as in Lemma 3.1 gives the stability estimate

(6.18) $\max \left(\left\|\vec{y}_{i}\right\|,\left|y_{i}\right|\right)$

$$
\leqslant \text { const }\left(|\bar{y}|+\max _{j=0(1)(i-1)}\left[\left(1+h_{j} t_{j+1 / 2}^{\alpha}\right)^{2}\left(h_{j} t_{j+1 / 2}^{\alpha}\left\|\vec{f}_{j}\right\|+\left|f_{j}\right|\right)\right]\right)
$$

which should be compared to (3.13). Now assume that $h_{j} t_{j+1 / 2}^{\alpha} \leqslant$ const holds on the whole interval of integration. Then we get stability for

$$
\begin{gathered}
\frac{y_{i j}-y_{i}}{h_{i}}=-\lambda \sum_{l=1}^{k} \hat{a}_{j l} t_{i l}^{\alpha} y_{i l}+t_{i m_{j}}^{\alpha} f_{i m_{j}}, \quad 1 \leqslant m_{j} \leqslant k, y_{0}=\bar{y}, \alpha>0, \\
\frac{y_{i+1}-y_{i}}{h_{i}}=-\lambda \sum_{l=1}^{k} \hat{b}_{l} t_{i l}^{\alpha} y_{i l}+t_{i m}^{\alpha} f_{i m}, \quad 1 \leqslant m \leqslant k
\end{gathered}
$$


by the following perturbation approach:

$$
\begin{aligned}
\frac{y_{i j}-y_{i}}{h_{i}}= & -\lambda t_{i+1 / 2}^{\alpha} \sum_{l=1}^{k} \hat{a}_{j l} y_{i l} \\
& -\lambda t_{i+1 / 2}^{\alpha}\left(\sum_{l=1}^{k} \hat{a}_{j l}\left(\frac{t_{i}^{\alpha}-t_{i+1 / 2}^{\alpha}}{t_{i+1 / 2}^{\alpha}}\right) y_{i l}+\frac{t_{i m_{j}}^{\alpha}}{t_{i+1 / 2}^{\alpha}} f_{i j}\right),
\end{aligned}
$$

$$
\begin{aligned}
\frac{y_{i+1}-y_{i}}{h_{i}}= & -\lambda t_{i+1 / 2}^{\alpha} \sum_{l=1}^{k} \hat{b}_{l} y_{i l} \\
& -\lambda t_{i+1 / 2}^{\alpha}\left(\sum_{l=1}^{k} \hat{b}_{l}\left(\frac{t_{i}^{\alpha}-t_{i+1 / 2}^{\alpha}}{t_{i+1 / 2}^{\alpha}}\right) y_{i l}+\frac{t_{i m}^{\alpha}}{t_{i+1 / 2}^{\alpha}} f_{i}\right) .
\end{aligned}
$$

Since $t_{i m_{j}}^{\alpha} / t_{i+1 / 2}^{\alpha} \leqslant$ const holds, estimate (5.18) gives

$$
\begin{aligned}
& \max \left(\left\|\vec{y}_{i}\right\|,\left|y_{i}\right|\right) \\
& \leqslant \operatorname{const}\left(|\bar{y}|+\max _{j}\left|\frac{t_{j}^{\alpha}-t_{j+1 / 2}^{\alpha}}{t_{j+1 / 2}^{\alpha}}\right| \max _{j=0(1)(i-1)} \max \left(\left\|\vec{y}_{j}\right\|,\left|y_{j}\right|\right)\right. \\
& \left.+\max _{j=0(1)(i-1)}\left(h_{j} t_{j+1 / 2}^{\alpha}\left\|\vec{f}_{j}\right\|+\left|f_{j}\right|\right)\right) .
\end{aligned}
$$

Choosing $h_{j}<\left((a-1) / \rho_{k}-\frac{1}{2}\right) t_{j}$, where $a=(1+\bar{\delta})^{1 / \alpha}$, we have

$$
\left|\left(t_{j}^{\alpha}-t_{j+1 / 2}^{\alpha}\right) / t_{j+1 / 2}^{\alpha}\right|<\bar{\delta} \text {. }
$$

Therefore if

(6.24) $h_{j} \leqslant \min \left(d_{0} t_{j}, d_{1} t_{j}^{-\alpha}\right), \quad d_{0}$ sufficiently small but independent of $t$ holds, we get the estimate

$$
\max \left(\left\|\vec{y}_{i}\right\|,\left|y_{i}\right|\right) \leqslant \operatorname{const}\left(|\vec{y}|+\max _{j=0(1)(i-1)}\left(h_{j} t_{j+1 / 2}^{\alpha}\left\|\vec{f}_{j}\right\|+\left|f_{j}\right|\right)\right)
$$

for the unique solution $y_{i j}, y_{i}$ of (6.19), (6.20). The stability consideration for the exponentially growing components are analogous to those following (3.25), (3.26). This stability result, obtained for scalar initial value problems can be extended to variable coefficient boundary value problems using the methods of Section 4.

In the sequel we assume that the function $f($ in $(5.11))$ fulfills

$$
f \in C^{k+1}\left(C_{x}\left(1, y^{*}(\infty)\right)\right.
$$

and that the assumptions on $f, b, S, y$ of Section 5 hold.

We define the local discretization error $t_{i j}^{\alpha} l_{i j}, t_{i+1 / 2}^{\alpha} l_{i}$ of $(6.2),(6.3),(6.4),(6.5)$ as

$$
\begin{gathered}
\frac{x\left(t_{i j}\right)-x\left(t_{i}\right)}{h_{i}}-\sum_{l=1}^{k} \hat{a}_{j l} t_{i l}^{\alpha} f\left(t_{i l}, x\left(t_{i l}\right)\right)=t_{i j}^{\alpha} l_{i j}, \\
\frac{x\left(t_{i+1}\right)-x\left(t_{i}\right)}{h_{i}}-\sum_{l=1}^{k} \hat{b}_{l} t_{i l}^{\alpha} f\left(t_{i l}, x\left(t_{i l}\right)\right)=t_{i+1 / 2}^{\alpha} l_{i},
\end{gathered}
$$


where $x(t)$ solves (5.11), (5.12), (5.13). A lengthy calculation (see Axelsson [3], Weiss [20]) gives

$$
\begin{gathered}
\left\|l_{i j}\right\| \leqslant \text { const } h_{i}^{k} \cdot \frac{1}{t_{i j}^{\alpha}}\left\|x^{(k+1)}\right\|_{\left[t_{i}, t_{i+1}\right]}, \\
\left\|l_{i}\right\| \leqslant \text { const } h_{i}^{k+1} \cdot \frac{1}{t_{i+1 / 2}^{\alpha}}\left\|x^{(k+2)}\right\|_{\left[t_{i}, t_{i+1}\right]}
\end{gathered}
$$

(6.30) holds because we only admit symmetric collocation points. We obtain

$$
\begin{gathered}
\left\|l_{i j}\right\| \leqslant \text { const } h_{i}^{k} t_{i}^{k \alpha} \exp \left(-\frac{\left(\lambda_{\min }-\delta\right)}{\alpha+1} t_{i}^{\alpha+1}\right), \quad t_{i} \geqslant \gamma, \\
\left\|l_{i}\right\| \leqslant \text { const } h_{i}^{k+1} t_{i}^{(k+1) \alpha} \exp \left(-\frac{\left(\lambda_{\min }-\delta\right)}{\alpha+1} t_{i}^{\alpha+1}\right), \quad t_{i} \geqslant \gamma,
\end{gathered}
$$

and

$$
\left\|l_{i j}\right\|=O\left(h_{i}^{k}\right), \quad\left\|l_{i}\right\|=O\left(h_{i}^{k+1}\right), \quad 1 \leqslant t_{i} \leqslant \gamma .
$$

The stability estimate (6.25) implies that we may equidistribute the functional

$$
h_{j} t_{j}^{\alpha}\left\|\vec{l}_{i}\right\|+\left\|l_{i}\right\| \leqslant \text { const } h_{i}^{k+1} t_{i}^{(k+1) \alpha} \exp \left(-\frac{\left(\lambda_{\min }-\delta\right)}{\alpha+1} t_{i}^{\alpha+1}\right), \quad t_{i} \geqslant \gamma,
$$

(where $\vec{l}_{i}=\left(l_{i 1}^{\prime}, \ldots, l_{i k}^{\prime}\right)^{\prime}$ holds) as long as (6.24) holds. Requiring a total accuracy $O(\varepsilon)$, we obtain bounds for the meshsizes

(a) $h_{i} \leqslant c_{0} \varepsilon^{1 /(k+1)}, \quad 1 \leqslant t_{i} \leqslant \gamma$,

$$
\text { (b) } h_{i} \leqslant \varepsilon^{1 /(k+1)} t_{i}^{-\alpha} \exp \left(\frac{\lambda_{\min }-\delta}{(k+1)(\alpha+1)} t_{i}^{\alpha+1}\right), \quad \gamma \leqslant t_{i} \leqslant T(\varepsilon) \text {, }
$$

where $T(\varepsilon)$ is defined in (2.32), (2.33). The number of meshpoints $N_{k}(\varepsilon) \sim$ const $1 / \varepsilon^{1 /(k+1)}$, the condition number of the linear set equations (6.2)-(6.5) $x_{k}\left(L_{h}\right)$ $\sim$ const $N_{k}(\varepsilon)$ (if equality holds in (6.35)), and we get

$$
\max _{\substack{i=0(1) N \\ j=1(1) k}} \max \left(\left\|y\left(t_{i j}\right)-y_{i j}\right\|,\left\|y\left(t_{i}\right)-y_{i}\right\|\right)=O(\varepsilon), \quad \varepsilon \rightarrow 0,
$$

for the locally unique solution $y_{i j}, y_{i}$ of (6.2), (6.3), (6.4), (6.5).

Using the superconvergence property of certain collocation schemes at the knots $t_{i}$, we can improve the bounds for the meshsizes (6.35). Let

$$
\omega(s)=\left(s-\rho_{1}\right)\left(s-\rho_{2}\right) \cdots\left(s-\rho_{k}\right) .
$$

We say that $\omega \in P_{r}, r>0$ if

$$
\int_{0}^{1} s^{i} \omega(s) d s=0, \quad i=0(1)(r-1) \text { and } \int_{0}^{1} s^{r} \omega(s) d s \neq 0
$$

holds. Obviously, $k+r-1$ is the order of accuracy of the integration formula

$$
\int_{0}^{1} \varphi(s) d s \approx \sum_{l=1}^{k} \hat{a}_{k l} \varphi\left(\rho_{l}\right)
$$

Applying the collocation scheme (6.2), (6.3) to the scalar initial value problem

$$
y^{\prime}=-\lambda y+f(t), \quad 1 \leqslant t, y(1)=\bar{y}, \lambda=\lambda_{\min }+i \lambda_{2},
$$


where (3.15) holds, we get for the global error $e_{i}=y\left(t_{i}\right)-y_{i}$ at the knots

$$
e_{i+1}=\gamma\left(-\lambda h_{i}\right) e_{i}+h_{i}\left(l_{i}-\lambda h_{i} \hat{b}^{\prime}\left(I-\lambda h_{i} \hat{A}\right)^{-1} \vec{l}_{i}\right), \quad e_{0}=0 .
$$

From Weiss [20] (or Axelsson [3]) we get

$$
\max \left(\left|l_{i}\right|,\left|h_{i} \hat{b}^{\prime}\left(I-\lambda h_{i} \hat{A}\right)^{-1} \vec{l}_{i}\right|\right) \leqslant \mathrm{const} h_{i}^{k+r}\left\|y^{(k+r+1)}\right\|_{\left[t_{i}, t_{i+1}\right]} .
$$

Using (6.16), we get

$$
\left|e_{i}\right| \leqslant \mathrm{const} \max _{j=0(1)(i-1)}\left(\left(1+h_{j}\right)^{2} h_{j}^{k+r}\left\|y^{(k+r+1)}\right\|_{\left[t_{j}, t_{j+1}\right]}\right) .
$$

For general $\alpha \geqslant 0$ we get, for the problem (3.1), (3.2),

$$
\left|e_{i}\right| \leqslant \mathrm{const} \max _{j=0(1)(i-1)} h_{j}^{k+r} \frac{1}{t_{j+1 / 2}^{\alpha}}\left\|y^{(k+r+1)}\right\|_{\left[t_{j}, t_{j+1}\right]}
$$

if $h_{j}$ fulfills (6.23).

Using the stability estimate (6.24) and proceeding as in Weiss [20], we obtain

THEOREM 6.1. Assume that $f(t, y) \in C^{k+r}\left(C_{x}\left(1, y^{*}(\infty)\right)\right)$, that the collocation scheme is $A$-stable and that $\omega \in P_{r}$. Then, for $\varepsilon$ sufficiently small, the collocation equations (6.2), (6.3), (6.4), (6.5) with

$$
\begin{aligned}
& \text { (a) } h_{j} \leqslant c_{0} \varepsilon^{1 /(k+1)}, \quad i \leqslant t \leqslant \gamma, \\
& \text { (b) } h_{j} \leqslant \varepsilon^{1 /(k+1)} t_{j}^{-\alpha} \exp \left(\frac{\lambda_{\min }-\delta}{(k+r)(\alpha+1)} t_{j}^{\alpha+1}\right), \quad \gamma \leqslant t_{j} \leqslant T(\varepsilon),
\end{aligned}
$$

where $T(\varepsilon)$ fulfills (2.33), have a locally unique solution $x_{i j}, x_{i}$ in a neighborhood of $y\left(t_{i j}\right), y\left(t_{i}\right)(y(t)$ solves $(5.1),(5.2),(5.3))$ and

$$
\begin{gathered}
\max _{i=0(1) N}\left\|y\left(t_{i}\right)-x_{i}\right\|=O(\varepsilon), \quad \varepsilon \rightarrow 0, \\
\max _{\substack{i=0(1) N \\
j=1(1) k}}\left\|y\left(t_{i j}\right)-y_{i j}\right\|=O\left(\varepsilon^{(k+1) /(k+r)}\right), \quad \varepsilon \rightarrow 0,
\end{gathered}
$$

holds. The Newton procedure for the collocation equations is quadratically convergent from a sphere of starting values which does not shrink as $\varepsilon \rightarrow 0$.

The number of necessary meshpoints $N_{k}(\varepsilon)$ fulfills

(6.47) $N_{k}(\varepsilon) \leqslant \varepsilon^{-1 /(k+r)}\left(\frac{k+r}{\lambda_{\min }-\delta} \exp \left(\frac{\lambda_{\min }-\delta}{k+r}\left(\varphi^{1 /(k+r)}-\frac{\gamma^{\alpha+1}}{\alpha+1}\right)\right)+\gamma-1\right)$, and the condition number of the linearized collocation equations $x_{k}\left(L_{h}\right) \sim$ const $N(\varepsilon), \varepsilon \rightarrow 0$ if equality holds in (6.44).

For Gauss points $r=k$ holds and the bound (6.44) is best possible.

7. A Numerical Test Problem. As a test problem we solve:

$$
\begin{gathered}
w^{(\text {iv })}=-1+\exp \left(-\frac{1}{2} w\right), \quad 1 \leqslant t \leqslant \infty, \\
w^{\prime \prime}(1)=0, \quad w^{\prime \prime \prime}(1)=1, \\
w \in C^{3}([1, \infty]),
\end{gathered}
$$

using the devised mesh-size sequences. This problem, which models the deflection of a pile embedded in soil was investigated by Lentini and Keller [10]. 
The obvious transformation

$$
y_{1}=w, \quad y_{2}=w^{\prime}, \quad y_{3}=w^{\prime \prime}, \quad y_{4}=w^{\prime \prime \prime} ; \quad y=\left(y_{1}, y_{2}, y_{3}, y_{4}\right)^{\prime}
$$

gives the four-dimensional problem

$$
\begin{aligned}
& y^{\prime}=f(y)=\left(\begin{array}{c}
y_{2} \\
y_{3} \\
y_{4} \\
-1+\exp \left(-\frac{1}{2} y_{1}\right)
\end{array}\right), \quad 1 \leqslant t<\infty \\
& {\left[\begin{array}{llll}
0 & 0 & 1 & 0 \\
0 & 0 & 0 & 1
\end{array}\right] y(1)=\left(\begin{array}{l}
0 \\
1
\end{array}\right) \text {, }} \\
& y \in C([1, \infty]) \text {. }
\end{aligned}
$$

The (only) stationary solution $y_{\infty}$ is given by

$$
y_{\infty}=(0,0,0,0)^{\prime},
$$

and the eigenvalues of the Jacobian $f_{y}\left(y_{\infty}\right)$ are

$$
\lambda_{l}=\left(\frac{1}{2}\right)^{1 / 4} e^{i(2 l-1) \pi / 4} ; \quad l=1,2,3,4
$$

so that

$$
\operatorname{Re} \lambda_{2}=\operatorname{Re} \lambda_{3}=-2^{-3 / 4}, \quad \operatorname{Re} \lambda_{1}=\operatorname{Re} \lambda_{4}=2^{-3 / 4}
$$

holds. Since the imaginary parts of $\lambda_{2,3}$ are nonzero, we have to expect (exponentially decaying) oscillating solutions.

We approximate (7.5), (7.6), (7.7) by the finite problem

$$
\begin{gathered}
x^{\prime}=f(x), \quad 1 \leqslant t \leqslant T(\varepsilon), \\
{\left[\begin{array}{llll}
0 & 0 & 1 & 0 \\
0 & 0 & 0 & 1
\end{array}\right] x(1)=\left(\begin{array}{l}
0 \\
1
\end{array}\right),} \\
{\left[\begin{array}{llll}
1 & 0 & 0 & 0 \\
0 & 1 & 0 & 0
\end{array}\right] x(T(\varepsilon))=\left(\begin{array}{l}
0 \\
0
\end{array}\right) .}
\end{gathered}
$$

The boundary condition (7.13) is admissible (i.e., it fulfills (2.29), (2.30)), however it is not optimal in the sense of (2.35).

Calculations using the optimal boundary condition have been performed by Lentini and Keller [10].

From (2.33) we get

$$
T(\varepsilon)=2^{3 / 4} \ln \frac{\varphi}{\varepsilon}
$$

where

$$
\varphi=\max _{t \geq 1}\left\|y(t) \exp \left(2^{-3 / 4} t\right)\right\|
$$

holds. Because (7.5) is autonomous and because all eigenvalues $\lambda_{l}$ are simple, no algebraic factors occur and $\delta$ (in (2.31)) can be set to zero. For the following computations the code COLSYS (see Ascher, Christiansen and Russell [1]) was used. All computations were performed on the CDC-CYBER 74 of the TU Vienna. An approximation to $\varphi$ was computed numerically by solving (7.11)-(7.13) using 7-stage 
collocation at Gaussian points with equidistant grid points and meshsize $h=0.2$. It turns out that $\varphi \approx 14$ holds. For $k$-stage collocation at Gaussian points we use the mesh-size sequence

$$
\begin{gathered}
h_{i}=\varepsilon^{1 / 2 k} \exp \left(\frac{2^{-7 / 4}}{k} t_{i}\right), \quad 1 \leqslant t_{i}<T(\varepsilon), \\
h_{N_{k}(\varepsilon)-1}=T(\varepsilon)-t_{N_{k}(\varepsilon)-1} .
\end{gathered}
$$

Again, $N_{k}(\varepsilon)$ denotes the number of meshpoints in [1,T(E)].

For the following calculations we choose $\varepsilon=10^{-2}, 10^{-3}, \ldots, 10^{-7}$ and $k=$ $1,2, \ldots, 6$. Table 1 lists $T(\varepsilon)$ (rounded). Obviously

$$
T\left(\frac{\varepsilon}{10}\right)=T(\varepsilon)+2^{3 / 4} \ln 10 \approx T(\varepsilon)+3.87
$$

holds. Tenfold accuracy is achieved by increasing the integration interval by 3.87 units.

Table 2 lists the number of meshpoints $N_{k}(\varepsilon)$ and the ratios

$$
\tau_{k}(\varepsilon)=N\left(\frac{\varepsilon}{10}, k\right) / N(\varepsilon, k)
$$

From (6.47) we conclude that

$$
\tau_{k}(\varepsilon) \approx 10^{1 / 2 k}
$$

holds. The values $10^{1 / 2 k}$ for $k=1, \ldots, 6$ are given in the last row of Table 2 , and it seems that the convergence of $\tau_{k}(\varepsilon)$ to $10^{1 / 2 k}$ (as $\left.\varepsilon \rightarrow 0\right)$ gets slower as $k$ increases. Table 1 also shows the enormous superiority of high order methods. For $k=1$ (midpoint rule) and $\varepsilon=10^{-7}, 7920$ grid points are required while for $k=7$ only 60 grid points are necessary in order to achieve (at least) the same accuracy (in fact Table 3 shows that the absolute error decreases for constant $\varepsilon$ and increasing $k$ )!

Table 3 contains the absolute errors

$$
e_{k}(\varepsilon)=\max _{i=0(1) N_{k}(\varepsilon)}\left\|y\left(t_{i}\right)-x_{i}(\varepsilon, k)\right\|
$$

(where $x_{i}(\varepsilon, k)$ solves the $k$-stage collocation equations (6.2)-(6.5) for (7.11)-(7.13)) and the corresponding (rounded) orders of convergence

$$
\ln \left(\frac{e_{k}(\varepsilon)}{e_{k}(\varepsilon / 10)}\right) / \ln 10
$$

Solutions for $k=1, \varepsilon=10^{-5}, 10^{-6}$ could not be obtained since the storage requirements were too large.

As mentioned before the absolute error for fixed $\varepsilon$ decreases as $k$ increases (except for $\varepsilon=10^{-4}, k=4,5$ ). This was also observed by Ascher and Weiss [2, Table 4.2] in the case of singular perturbation problems.

The orders of convergence are even for large $\varepsilon$ fairly accurate for $k=1,2$. For larger $k$ and large $\varepsilon$ convergence is faster (than order one in $\varepsilon$ ) but as $\varepsilon$ decreases the orders apparently converge to one. 
TABLE 1

\section{Integration intervals}

\begin{tabular}{c|llllll}
$\varepsilon$ & $10^{-2}$ & $10^{-3}$ & $10^{-4}$ & $10^{-5}$ & $10^{-6}$ & $10^{-7}$ \\
\hline$T(\varepsilon)$ & 12.18 & 16.06 & 19.93 & 23.8 & 27.67 & 31.54
\end{tabular}

TABLE 2

Number of mesh points and ratios

\begin{tabular}{|l|c|c|c|c|c|c|c|c|c|c|c|c|}
\hline$\varepsilon k$ & \multicolumn{2}{|c|}{1} & \multicolumn{2}{|c|}{2} & \multicolumn{2}{|c|}{3} & \multicolumn{2}{|c|}{4} & \multicolumn{2}{c|}{5} \\
\hline & $\mathrm{N}_{1}(\varepsilon)$ & $\tau_{1}(\varepsilon)$ & $\mathrm{N}_{2}(\varepsilon)$ & $\tau_{2}(\varepsilon)$ & $\mathrm{N}_{3}(\varepsilon)$ & $\tau_{3}(\varepsilon)$ & $\mathrm{N}_{4}(\varepsilon)$ & $\tau_{4}(\varepsilon)$ & $\mathrm{N}_{5}(\varepsilon)$ & $\tau_{5}(\varepsilon)$ & $\mathrm{N}_{6}(\varepsilon)$ & $\tau_{6}(\varepsilon)$ \\
\hline $10^{-2}$ & 27 & 3.04 & 17 & 1.88 & 15 & 1.67 & 14 & 1.57 & 14 & 1.5 & 14 & 1.43 \\
\hline $10^{-3}$ & 82 & 2.91 & 32 & 1.78 & 25 & 1.52 & 22 & 1.45 & 21 & 1.38 & 20 & 1.35 \\
\hline $10^{-4}$ & 253 & 3.03 & 57 & 1.81 & 38 & 1.53 & 32 & 1.38 & 29 & 1.34 & 27 & 1.33 \\
\hline $10^{-5}$ & 794 & 3.14 & 103 & 1.78 & 58 & 1.52 & 44 & 1.43 & 39 & 1.33 & 36 & 1.31 \\
\hline $10^{-6}$ & 2503 & 3.16 & 183 & 1.78 & 88 & 1.49 & 63 & 1.38 & 52 & 1.33 & 47 & 1.28 \\
\hline $10^{-7}$ & 7920 & & 326 & & 131 & & 87 & & 69 & & 60 & \\
\hline $10^{1 / 2 \mathrm{k}}$ & & 3.16 & & 1.78 & & 1.47 & & 1.33 & & 1.26 & & 1.21 \\
\hline
\end{tabular}

TABLE 3

Errors and orders of convergence

\begin{tabular}{|c|c|c|c|c|c|c|c|c|c|c|}
\hline$\varepsilon$ & \multicolumn{2}{|c|}{1} & \multicolumn{2}{c|}{2} & \multicolumn{2}{c|}{3} & \multicolumn{2}{c|}{4} & \multicolumn{2}{c|}{5} \\
\hline \multirow{2}{*}{$1 . \mathrm{E}-2$} & $3.23 \mathrm{E}-3$ & 1.0 & $2.68 \mathrm{E}-4$ & 1.6 & $2.24 \mathrm{E}-4$ & 2.8 & $2.20 \mathrm{E}-4$ & 2.8 & $8.00 \mathrm{E}-5$ & 2.5 \\
\hline & $\mathrm{e}_{1}(\varepsilon)$ & & $\mathrm{e}_{2}(\varepsilon)$ & & $\mathrm{e}_{3}(\varepsilon)$ & & $\mathrm{e}_{4}(\varepsilon)$ & & $\mathrm{e}_{5}(\varepsilon)$ & \\
\hline $1 . \mathrm{E}-3$ & $3.03 \mathrm{E}-4$ & 1.0 & $5.60 \mathrm{E}-6$ & 1.0 & $3.13 \mathrm{E}-7$ & 1.7 & $2.84 \mathrm{E}-7$ & 1.9 & $2.84 \mathrm{E}-7$ & 1.8 \\
\hline $1 . \mathrm{E}-4$ & $3.05 \mathrm{E}-5$ & & $5.55 \mathrm{E}-7$ & 1.0 & $6.00 \mathrm{E}-9$ & 0.8 & $3.30 \mathrm{E}-9$ & 0.8 & $3.79 \mathrm{E}-9$ & 1.4 \\
\hline $1 . \mathrm{E}-5$ & & & $5.70 \mathrm{E}-8$ & 1.0 & $1.00 \mathrm{E}-9$ & 1.1 & $5.0 \mathrm{E}-10$ & 1.2 & $1.45 \mathrm{E}-10$ & 1.1 \\
\hline $1 . \mathrm{E}-6$ & & & $5.00 \mathrm{E}-9$ & & $8.2 \mathrm{E}-11$ & & $3.0 \mathrm{E}-11$ & & $1.20 \mathrm{E}-11$ & \\
\hline
\end{tabular}

Acknowledgement. The authors would like to thank Professor Uri Ascher (UBC) for many helpful discussions which led to a major improvement of the paper.

Department of Mathematics

The University of Texas at Austin

RLM 8.100

Austin, Texas 78712

Institut fuer Angewandte Mathematik

Technische Universität Wien

Gusshausstrasse 27-29

A-1040 Wien, Austria 
1. U. AsCheR, J. Christiansen \& R. D. Russell, "A collocation solver for mixed order systems of boundary value problems," Math. Comp., v. 33, 1979, pp. 659-679.

2. U. AsCheR \& R. Weiss, Collocation for Singular Perturbation Problems I: First Order Systems with Constant Coefficient, UBC Technical Report, 1981; also to appear in SIAM J. Numer. Anal.

3. O. AXELSSON, "A class of $A$-stable methods," $B I T$, v. 9, 1969, pp. 185-199. MR 40 \#266.

4. C. DE Boor \& B. SwarTZ, "Collocation at Gaussian points," SIAM J. Numer. Anal., v. 10, 1973, pp. 582-606.

5. C. DE BOOR \& R. WEISS, “"SOLVEBLOCK”-A package for almost block diagonal linear systems," ACM Trans. Math. Software, v. 61, 1980, pp. 80-87.

6. F. R. DE HOOG \& R. WeISs, "The numerical solution of boundary value problems with an essential singularity," SIAM J. Numer. Anal., v. 10, 1979, pp. 637-669.

7. F. R. DE HOOG \& R. WeIss, "On the boundary value problem for systems of ordinary differential equations with a singularity of the second kind," SIAM J. Math. Anal., v. 11, 1980, pp. 41-60.

8. F. R. DE HOOG \& R. WeISs, "An approximation method for boundary value problems on infinite intervals," Computing, v. 24, 1980, pp. 227-239.

9. H. Keller, "Approximation methods for nonlinear problems with application to two-point boundary value problems," Math. Comp., v. 29, 1975, pp. 464-474.

10. M. LeNTINI \& H. B. Keller, "Boundary value problems on semi-infinite intervals and their numerical solution," SIAM J. Numer. Anal., v. 17, 1980, pp. 577-604.

11. M. Lentini \& V. Pereyra, "An adaptive finite difference solver for nonlinear two-point boundary value problems with mild boundary layers," SIAM J. Numer. Anal., v. 14, 1977, pp. 99-111.

12. P. A. Markowich, Analysis of Boundary Value Problems on Infinite Intervals, MRC TSR \#2138, 1980; also to appear in SIAM J. Math. Anal.

13. P. A. MARKowich, "A theory for the approximation of solutions of boundary value problems on infinite intervals," SIAM J. Math. Anal., v. 13, 1982, pp. 484-513.

14. P. A. MARKowich, Eigenvalue Problems on Infinite Intervals, MRC TSR \#2157, 1980; to appear in this Journal.

15. J. B. MCLeod, “Von Karman's swirling flow problem," Arch. Rational Mech. Anal., v. 1, 1969, pp. 91-102.

16. C. A. RINGhofer, On Collocation Methods for Singularly Perturbed Boundary Value Problems, Thesis, TU Vienna, Austria, 1981.

17. R. D. Russell, "Collocation for systems of boundary value problems," Numer. Math., v. 23, 1974, pp. 119-133.

18. R. D. Russell \& J. Christiansen, "Adaptive mesh selection strategies for solving boundary value problems," SIAM J. Numer. Anal., v. 15, 1978, pp. 59-80.

19. W. SCHNEIDER, "A similarity solution for combined forced and free convection flow over a horizontal plate," Internat. J. Stat. and Mass Transfer, v. 22, 1979, pp. 1401-1406.

20. R. Weiss, "The application of implicit Runge-Kutta and collocation methods to boundary value problems," Math. Comp., v. 28, 1974, 449-464. 